\title{
An observable signature of a background deviating from Kerr
}

\author{
Georgios Lukes-Gerakopoulos, ${ }^{1,2}$ Theocharis A. Apostolatos, ${ }^{2}$ and George Contopoulos ${ }^{1}$ \\ ${ }^{1}$ Academy of Athens, Research Center for Astronomy, \\ Soranou Efesiou 4, GR-11527, Athens, GREECE \\ ${ }^{2}$ Section of Astrophysics, Astronomy, and Mechanics, Department of Physics, \\ University of Athens, Panepistimiopolis Zografos GR15783, Athens, Greece
}

\begin{abstract}
By detecting gravitational wave signals from extreme mass ratio inspiraling sources (EMRIs) we will be given the opportunity to check our theoretical expectations regarding the nature of supermassive bodies that inhabit the central regions of galaxies. We have explored some qualitatively new features that a perturbed Kerr metric induces in its geodesic orbits. Since a generic perturbed Kerr metric does not possess all the special symmetries of a Kerr metric, the geodesic equations in the former case are described by a slightly nonintegrable Hamiltonian system. According to the Poincaré-Birkhoff theorem this causes the appearance of the so-called Birkhoff chains of islands on the corresponding surfaces of section in between the anticipated KAM curves of the integrable Kerr case, whenever the intrinsic frequencies of the system are at resonance. The chains of islands are characterized by finite width, i.e. there is a finite range of initial conditions that correspond to a particular resonance and consequently to a constant rational ratio of intrinsic frequencies. Thus while the EMRI changes adiabatically by radiating energy and angular momentum, by monitoring the frequencies of a signal we can look for a transient pattern, in the form of a plateau, in the evolution of their ratio. We have shown that such a plateau is anticipated to be apparent in a quite large fraction of possible orbital characteristics if the central gravitating source is not a Kerr black hole. Moreover the plateau in the ratio of frequencies is expected to be more prominent at specific rational values that correspond to the strongest resonances. This gives a possible observational detection of such non-Kerr exotic objects.
\end{abstract}

PACS numbers: 04.30.-w; 97.60.Lf; 05.45.-a

Keywords: Gravitational waves, black holes, KAM theorem

\section{INTRODUCTION}

The enterprize of searching for gravitational waves with the interferometric devices that are already operating in various places around the globe is under way. Unfortunately no positive outcome has come up yet [1]. Gravitational waves visiting us from powerful cosmic sources are very weak upon reaching our planet. The sensitivity of the present day detectors is not adequate to distinguish undoubtedly the signal from noise; it is improving continuously though. On the other hand null detection of gravitational waves puts firm constraints on various physical parameters related with known sources of gravitational waves (e.g. rotating neutron stars [2]).

LISA, the future interferometric detector that is planned to be launched by NASA and ESA during the forthcoming decade, will be much more sensitive in detecting gravitational waves from much more massive and much more distant sources [3]. Among such sources are EMRIs (extreme-mass-ratio inspirals), that is low mass compact objects (neutron stars or solar-mass black holes) trapped in the gravitational field of supermassive compact objects which are probably located at the centers of galaxies [4 $[6]$. According to conventional astrophysical wisdom, such supermassive objects are Kerr black holes. The gravitational waves emitted in a Kerr background have been extensively studied by a large number of people, and useful techniques have been invented to gain physical information about the characteristics of these sources from the analysis of the corresponding waveforms [ 7 . Of course the extraction of valuable astrophysical information from the gravitational waves will be hindered by instrumental noise [3] and confusion noise [10] (background unresolved signals). Furthermore practical difficulties concerning the search in the multi-parametric space of templates for EMRIs that will be used to detect such signals render the accomplishment of such a goal even harder [11].

EMRIs are ideal sources of gravitational waves through which one could explore the background metric that the lighter object is tracing. In 1995 Ryan [12, 13] showed that almost circular and almost equatorial orbits in a generic stationary and axisymmetric background could reveal the lower order mass moments and mass-current moments by monitoring the corresponding evolution of gravitational waves that are emitted in the weak field region. Since all multiple moments of a Kerr black hole are determined only by its mass and its spin, one needs to know at least the quadrupole moment (besides the mass and the spin) of the central object to decide if the object is a Kerr metric or not. However, while the measurement of the quadrupole moment is in principle feasible with LISA, the accuracy of such measurement is limited due to a number of reasons: (i) The orbits of EMRIs are expected to be much more general than simply circular and equatorial. (ii) The analysis is limitted to work in the weak field region, where the 
gravitational wave emission is not as intense as in the strong field region. (iii) The source should not be a very distant one, so that the signal to noise ratio in the analysis of gravitational waves is sufficiently high to be able to obtain the parameters of the source with good accuracy.

An alternative approach for checking whether the background metric is that of a Kerr black hole is to focus on a characteristic signature of EMRIs in a generic non-Kerr background. This could be a yes/no measurement from which one could decide if the field into which the small object orbits is that of a massive Kerr black hole or not. Various studies have been performed towards this line of thought. Collins and Hughes [14] built a perturbed Kerr metric and explored the effect of these perturbations on physically observable quantities related with geodesics in such a background. Later, Glampedakis and Babak [8] showed that a perturbed Kerr metric leads to a significant mismatch between the corresponding waveforms, although there is an issue of confusion between waveforms of the specific nonKerr metric and a Kerr metric with different orbital parameters. A similar approach assuming a more astrophysically oriented non-Kerr metric was followed by Barausse et al [15]. Recently, Gair et al [16] used a specific exact solution of vacuum Einstein equations, namely the Manko-Novikov solution [17], which could be turned into a pure Kerr metric by dialing a single parameter, and studied the geodesics in such a metric. Their analysis showed that for a range of metric parameters there are two ring-like regions of bounded orbits in this space-time. In the outer one the orbits look regular, as if there exists an isolating integral of motion, while in the inner region the orbits seem to be ergodic. The two regions, though, merge for a range of orbital parameters. It was suggested by Gair et al [16] that the transition of the orbits from the regular to the ergodic region when the two aforementioned regions merge, could have a clear observable effect on the frequency spectrum of the corresponding gravitational waves, especially if this transition is repetitive from the former to the latter region and vice versa. Another effect that was thoroughly explored by Gair et al is the instability that may arise just before the orbiting body arrives at its innermost circular orbit (ISCO). This effect might have a strong influence on the gravitational waveform at the final stage of the corresponding EMRI. However, these new orbital features vary significantly with the orbital and metric parameters.

Thus it would be important to seek for a generic feature that clearly distinguishes a non-Kerr metric from a Kerr one. Then it would be much easier to perform a specialized observation to measure it. The fact that Kerr black holes are so perfect and symmetric objects which are created naturally, renders all other gravitational fields of isolated massive objects quite different. In contrast to the Kerr metric, any other generic solution of Einstein field equations that describes the neighborhood of an isolated massive object is expected to be less symmetric. Even axisymmetric and stationary metrics, but otherwise generic ones, are not expected to have something analogous to the Carter Killing tensor field, as it happens with the Kerr metric [18]. Therefore the existence of a Carter-like constant that makes the geodesic orbits in a Kerr metric to be characterized by a completely integrable system of equations [19], most probably is lost in a non-Kerr axially symmetric and stationary, asymptotically flat metric. Geodesic orbits in such a generic metric is described by a nonintegrable Hamiltonian that deviates from the corresponding integrable Kerr-type Hamiltonian. Furthermore, if such a non-Kerr metric is not drastically different from a Kerr one, a generic axially symmetric, and stationary metric, that could in principle be realized by natural processes, could be considered as a perturbed Kerr metric.

The KAM theorem of Kolmogorov, Arnold and Moser [20], applies to Hamiltonian systems that are slightly perturbed from integrable systems. According to this general theorem most tori of the corresponding integrable system, that is the toroidal hyper-surfaces of phase-space on which the phase orbits of the system are lying, get slightly deformed in the perturbed system, but they are not destroyed. This is exactly the case presented by Gair et al [16], who studied geodesic orbits in the so-called Manko-Novikov metric.

On the other hand according to the Poincaré-Birkhoff theorem, the resonant tori (the tori characterized by a rational ratio of winding frequencies) of the integrable system disintegrate when the system gets perturbed, and consequently a chain of islands is formed on a surface of section, instead of an infinite number of sets of periodic points which is a characteristic feature of an integrable system. These islands are characterized, as in the resonant tori of the integrable system, by a ratio of winding frequencies of the system that equals a rational number. The appearance of such islands is a very distinct new signature of a slightly nonintegrable system and it is qualitatively different from the behavior of geodesics in a Kerr spacetime. They are always present independently of the way the system deviates from the corresponding integrable one. This new feature, if observable, could clearly distinguish any kind of perturbed Kerr metric from a pure Kerr metric [21].

We start by showing that these Birkhoff islands are actually present in the Manko-Novikov metric studied in [16]. However, such islands were not found in [16], because a very thorough exploration of the phase-space is needed in order to reveal their existence. The rotation number, a tool widely used in the study of systems where chaos and order coexist, is employed in the search of suitable initial orbital conditions that lead to the formation of Birkhoff islands on a surface of section. The rotation number is an index that is directly related to observable quantities (see below).

Next we argue that, since these islands can be identified by monitoring specific quantities, namely the frequencies of the emitted gravitational waves, we can tell if the metric in which the orbiting object is moving is a Kerr or a non-Kerr 
one by not observing or observing, respectively, a fixed ratio of the two polar frequencies for a period of time while the frequencies themselves are evolving. An observed plateau in the evolution of the ratio of the corresponding frequencies would be an unambiguous signature of a non-Kerr metric. Moreover since the most pronounced resonances (thicker Birkhoff islands) correspond to simple-integer-number ratios, like $2: 3$, or $1: 2$, the value of the ratio of frequencies that is related to the most extended plateaus should be a simple-integer-number ratio as well. Thus we can focus our signal analysis on the time interval when the ratio of polar frequencies assumes that value and investigate the existence or not of a plateau in the evolution of the ratio of frequencies. Even a null result in such an analysis can be used to put firm constraints in the likelihood that we have observed an EMRI in a pure Kerr metric through gravitational waves.

Now from a practical point of view, we argue that a large fraction of EMRIs that get trapped in the gravitational field of a supermassive compact object in a generic eccentric and non-equatorial orbit will evolve in such a way that the corresponding orbit will eventually cross a resonant torus (if the central massive object is a Kerr black hole) or the corresponding Birkhoff chain of islands (if the central object is described by a perturbed Kerr metric). Respectively, the ratio of the frequencies will vary strictly monotonically, or it will form a plateau. In the latter case the duration of the plateau will depend (i) on the particular metric which is related to the characteristics of the central massive object, (ii) on the orbital parameters of the low-mass object, and (iii) on the ratio of the masses involved in the binary as well. This plateau can in principle be identified for a source with a sufficiently high signal-to-noise ratio.

The present paper is organized as follows. In Section 2 we repeat the basic characteristics of the Manko-Novikov solution which was used and thoroughly analyzed in [16] as an example of a non-Kerr metric which could be transformed into a Kerr one by suitably adjusting a single parameter. We are using the same metric to exhibit the new feature that we propose to use as a yes/no test of a Kerr metric. In Sec. 3 we start with a short description of the Poincaré-Birkhoff theorem as a theoretical basis applied in the perturbed Kerr case, and then we present a thorough study of the surfaces of section of the specific non-Kerr metric; the Manko-Novikov one. We show how we have determined the initial conditions so as to form a few Birkhoff chains of islands, by implementing a numerical measurement of the rotation number for each geodesic. Both regions of bound orbits that are present in this metric are thoroughly explored through surfaces of section and the final picture is discussed in every case; either when the two regions are separated or connected. Apart from the Birkhoff chains of islands, a new characteristic found in our analysis is the existence of regular non-chaotic orbits in the inner region. In Section 4 we adiabatically change the parameters of the geodesic orbits (imitating the dissipative behavior of gravitational waves) so that the phase orbit crosses a chain of Birkhoff islands. Here we present a quantitative result that relates the duration of the plateau with the various characteristics of the central and of the orbiting bodies. We study the possible ways that this crossing could take place and we get a crude estimate of the range of orbital parameters of EMRIs that will eventually lead the system to pass through a Birkhoff chain of islands of a strong resonance. In Section 5 we analyze the evolution of the ratio of frequencies before, during, and after the crossing of a resonance by the non-geodesic orbit and we obtain a plateau in the ratio of the monitored frequencies during the crossing. We close our paper with Section 6 by summarizing the physical conclusions drawn from our analysis. Finally in Appendix $\mathrm{A}$ we give the relations between the Boyer-Lindquist coordinates and the Keplerian elements, and in Appendix B we introduce a new set of variables, which are more appropriate for our Fourier analysis.

\section{THE MANKO-NOVIKOV METRIC}

\subsection{Description of the MN spacetime}

In order to demonstrate how the aforementioned new features appear and what are their consequences, we are going to use the same type of non-Kerr metric which was used by Gair et al [16]. It is a subclass of the multiparametric family of spacetimes that Manko and Novikov constructed in 1992 [17] as a generalization of the Kerr (and the Kerr-Newman) metric in an attempt to describe the gravitational field of an arbitrary rotating and axially symmetric isolated object. The corresponding construction, which is an exact solution of the vacuum Einstein equations, was achieved by a non-linear superposition of the Kerr spacetime with an arbitrary static vacuum Weyl field in a concise analytical form (see 22]). While the general solution of Manko and Novikov does depend on an infinite number of parameters that are related to the multipole moments of the gravitational field, the particular subclass that we are going to study (the one studied in [16] as well) depends on three parameters. Two of them determine the mass $M$ and the spin $S$ of the source of the field, while the third one, $q$, is a dimensionless index that measures the deviation of its quadrupole mass moment from the quadrupole moment of the corresponding Kerr black hole (a Kerr metric that has the mass and the spin of the new metric). Thus the first four non-zero mass and current-mass moments $M_{l}, S_{l}$ 
(with $l \leq 3$ ) are fully characterized by these three parameters:

$$
\begin{array}{ll}
M_{0}=M, & S_{1}=S, \\
M_{2}=-M\left[\left(\frac{S}{M}\right)^{2}+q M^{2}\right], & S_{3}=-M\left[\left(\frac{S}{M}\right)^{3}+2 q M^{2}\left(\frac{S}{M}\right)\right],
\end{array}
$$

while the higher order moments are in general higher order polynomials with respect to $q$. We have chosen to present the moments in this way so that the first part of each moment is merely the moment of the corresponding Kerr metric. We remind that all multiple moments of a Kerr metric are characterized by two parameters $M$ and $S / M$ and are given by the following concise formula:

$$
M_{l}+\imath S_{l}=M\left(\imath \frac{S}{M}\right)^{l} .
$$

Having these in mind, we proceed to write the analytical expressions for the metric functions of the particular subclass of Manko-Novikov metric (hereafter called MN metric). As for all stationary, axially symmetric, and mirror symmetric vacuum spacetimes the Weyl-Papapetrou line element for this metric is

$$
d s^{2}=-f(d t-\omega d \phi)^{2}+f^{-1}\left[e^{2 \gamma}\left(d \rho^{2}+d z^{2}\right)+\rho^{2} d \phi^{2}\right]
$$

where all metric functions $f, \omega, \gamma$ should be considered as functions of the prolate spheroidal coordinates $x, y$ (the coordinates $\rho, z$ are the corresponding cylindrical coordinates which could be expressed as functions of $x, y$ as well). Thus

$$
\rho=k \sqrt{\left(x^{2}-1\right)\left(1-y^{2}\right)}, \quad z=k x y
$$

and

$$
\begin{aligned}
& f=e^{2 \psi} \frac{A}{B}, \\
& \omega=2 k e^{-2 \psi} \frac{C}{A}-4 k \frac{\alpha}{1-\alpha^{2}}, \\
& e^{2 \gamma}=e^{2 \gamma^{\prime}} \frac{A}{\left(x^{2}-1\right)\left(1-\alpha^{2}\right)^{2}} \text {, } \\
& A=\left(x^{2}-1\right)(1+a b)^{2}-\left(1-y^{2}\right)(b-a)^{2} \text {, } \\
& B=[(x+1)+(x-1) a b]^{2}+[(1+y) a+(1-y) b]^{2} \text {, } \\
& C=\left(x^{2}-1\right)(1+a b)[(b-a)-y(a+b)] \\
& +\left(1-y^{2}\right)(b-a)[(1+a b)+x(1-a b)] \text {, } \\
& \psi=\beta \frac{P_{2}}{R^{3}}, \\
& \gamma^{\prime}=\ln \sqrt{\frac{x^{2}-1}{x^{2}-y^{2}}}+\frac{3 \beta^{2}}{2 R^{6}}\left(P_{3}^{2}-P_{2}^{2}\right) \\
& +\beta\left(-2+\sum_{\ell=0}^{2} \frac{x-y+(-1)^{2-\ell}(x+y)}{R^{\ell+1}} P_{\ell}\right), \\
& a=-\alpha \exp \left[-2 \beta\left(-1+\sum_{\ell=0}^{2} \frac{(x-y) P_{\ell}}{R^{\ell+1}}\right)\right], \\
& b=\alpha \exp \left[2 \beta\left(1+\sum_{\ell=0}^{2} \frac{(-1)^{3-\ell}(x+y) P_{\ell}}{R^{\ell+1}}\right)\right] \text {, } \\
& R=\sqrt{x^{2}+y^{2}-1} \text {, } \\
& P_{\ell}=P_{\ell}\left(\frac{x y}{R}\right) \text {, }
\end{aligned}
$$

where $P_{\ell}(z)$ denotes the Legendre polynomial of order $l$ given by

$$
P_{\ell}(z)=\frac{1}{2^{\ell} \ell !}\left(\frac{d}{d z}\right)^{\ell}\left(z^{2}-1\right)^{\ell} .
$$


The three parameters $k, \alpha, \beta$ that appear in the formulae above are the three parameters that characterize the metric and are related to the mass $M$, the spin $S$, and the quadrupole deviation $q$ through the following expressions:

$$
\alpha=\frac{-M+\sqrt{M^{2}-(S / M)^{2}}}{(S / M)}, k=M \frac{1-\alpha^{2}}{1+\alpha^{2}}, \beta=q\left(\frac{1+\alpha^{2}}{1-\alpha^{2}}\right)^{3} .
$$

The formula for $\alpha$ has been written as a function of $M$ and $(S / M)$ (both of them have dimensions of mass), which are the physical parameters of the central object that are common in the MN metric and the corresponding Kerr metric. In [16] the formula for $\alpha$ is written as a function of the dimensionless spin parameter $\chi=S / M^{2}$. We use the parameter $\chi$ later in our numerical examples for simplicity.

The Kerr metric is a limiting case of the MN metric for $q=0$. A non-zero $q$ parameter distinguishes the two types of metrics. At this point we should note that the MN metric is the general solution described in [17] with all $a_{i}$ parameters set to zero except of $a_{2}$ which is the parameter $\beta$ that shows up in the metric functions. Also, the -2 term in Eq. (5h) has been moved outside the sum (c.f., Eq. (3h) of [16]) so as to avoid any confusion.

According to the thorough analysis of [16] the MN metric is not a black hole solution, in agreement with the no-hair theorem. The central singularity is surrounded by a horizon which is broken along the equator by a circular line singularity. As is the case with the Kerr metric, the MN spacetime possesses an ergoregion (the region between the static limit $g_{t t}=0$ and the event horizon $\left(g_{x x}\right)^{-1}=0$ ) in the form of lobes that surround the horizon. Moreover a region of closed time-like curves (where $g_{\phi \phi}<0$ ) is present (when $q \neq 0$ ) in the form of lobes that overlap with the ergoregion. In all cases we have investigated the region of permitted motion is not overlapping with the region of the closed time-like curves, even though their borders may touch each other.

Closing this general description of the MN metric we should emphasize once more that this metric is an exact vacuum solution that can be continuously turned into a Kerr metric by setting $q=0$. Thus the MN metric is a good candidate to describe the field of a stationary axisymmetric and isolated spinning object (the multipole moments of which differentiate the corresponding metric from a Kerr one) outside some central region where singular behavior of the metric functions shows up.

\subsection{Geodesics in the MN spacetime}

Working in Lagrangian formulation, the geodesic orbits in the MN metric are described as equations of motion of the following Lagrangian

$$
L=\frac{1}{2} \mu g_{\mu \nu} \dot{x}^{\mu} \dot{x}^{\nu}
$$

where $\mu$ is the rest mass of the orbiting body and ${ }^{\cdot} \equiv \frac{d}{d \tau}\left(\tau\right.$ denotes the proper time along the orbit: $\left.(d \tau)^{2}=-(d s)^{2}\right)$. Such a Lagrangian of purely kinetic form is invariant along the orbit. Its invariance is related to the fact that the rest mass of the orbiting test body is a conserved quantity $\left(p^{\nu} p_{\nu}=-\mu^{2}\right)$, and thus the value of $L$ is $-\mu / 2$. Due to stationarity and axisymmetry of the MN metric there are two more integrals of motion, respectively; the specific energy

$$
E=-\frac{p_{t}}{\mu}=f(\dot{t}-\omega \dot{\phi})
$$

and the specific $z$-component of angular momentum of the orbiting test body

$$
L_{z}=\frac{p_{\phi}}{\mu}=f \omega(\dot{t}-\omega \dot{\phi})+\rho^{2} \dot{\phi} / f
$$

("specific" means per unit rest mass). By suitable linear combinations of these integrals of motion one yields the following expressions for $\dot{\phi}$ and $\dot{t}$ :

$$
\begin{aligned}
\dot{\phi} & =\frac{f}{\rho^{2}}\left(L_{z}-\omega E\right), \\
\dot{t} & =\frac{\omega f}{\rho^{2}}\left[L_{z}+\omega E\left(\frac{\rho^{2}}{\omega^{2} f^{2}}-1\right)\right] .
\end{aligned}
$$

The two remaining equations of motion that determine $\rho(\tau)$ and $z(\tau)$ are thus sufficient to fully describe a geodesic orbit in such a spacetime; the $\phi$ and $t$ coordinates are subsequently obtained by direct integration of Eqs. (1112). 
Substituting the above expressions in the Lagrangian of Eq. (8) and taking into account the constant value of the Lagrangian itself, we obtain the following constraint between the remaining four coordinates $(\rho, z, \dot{\rho}, \dot{z})$ of phase space

$$
\frac{1}{2}\left(\dot{\rho}^{2}+\dot{z}^{2}\right)+V_{\text {eff }}(\rho, z)=0
$$

where

$$
V_{\mathrm{eff}}(\rho, z)=\frac{1}{2} e^{-2 \gamma}\left[f-E^{2}+\left(\frac{f}{\rho}\left(L_{z}-\omega E\right)\right)^{2}\right]
$$

plays the role of an effective 2-dimensional potential. Note that this expression is somewhat different from the expression for the effective potential in Eq. (13) of [16]; the present expression is written so as to resemble better the newtonian analogue of energy conservation in potential wells. The curve along which $V_{\text {eff }}=0$ determines the region of allowed orbits in the polar plane (the $(\rho, z)$ plane that rotates along with the orbiting body). The orbits can only move in the interior of such a curve, since the effective potential is negative inside it (if we take into account the third spatial coordinate $\phi$ as well it is actually a toroidal space-like surface centered at the central singularity). Whenever an orbit reaches the $V_{\text {eff }}=0$ curve both velocities $\dot{\rho}, \dot{z}$ become equal to zero. So we will denote this curve as the curve of zero-velocity (CZV).

(a)

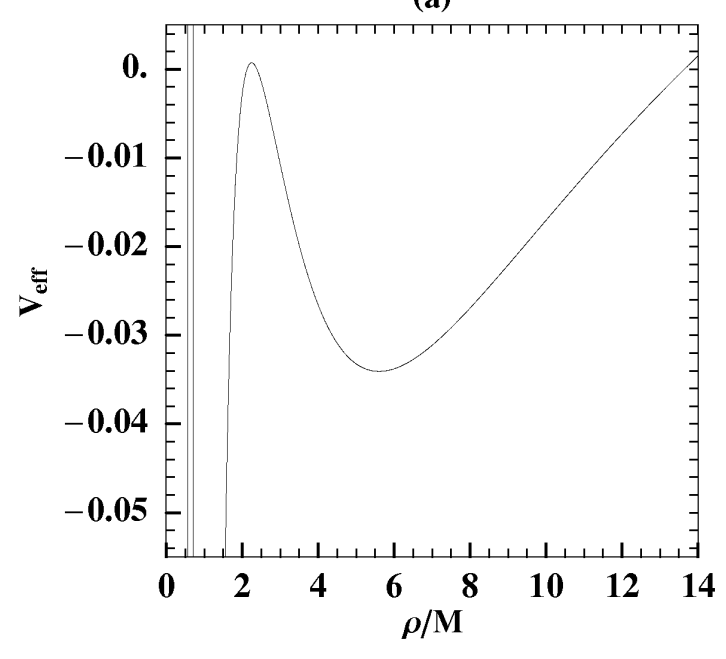

(b)

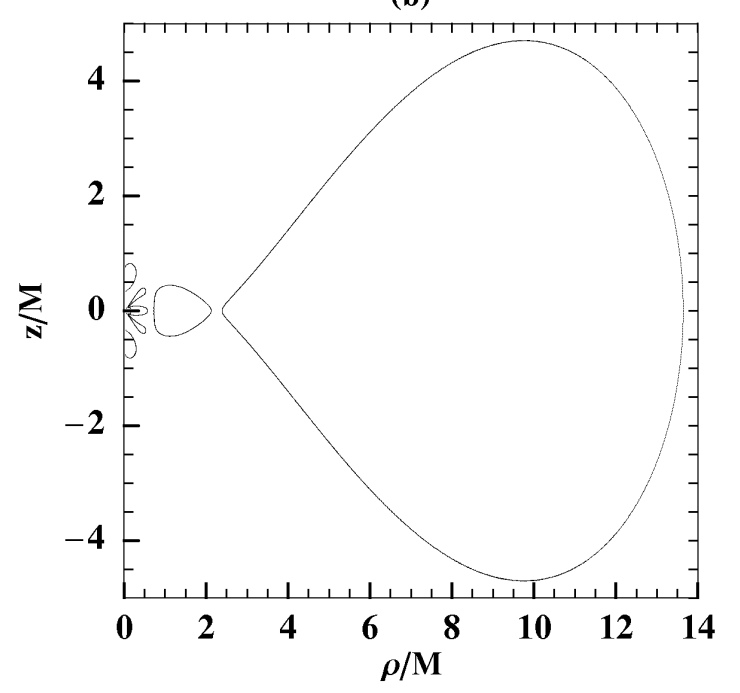

FIG. 1: (a) The shape of the 2-dimensional well $V_{\text {eff }}(\rho, z)$ near $V_{\text {eff }}(\rho, z)=0$ along the line $z=0$ for the MN metric with parameters $\chi=0.9, q=0.95$ and orbital parameters $E=0.95, L_{z}=3 M$. (b) The CZVs $\left(V_{\text {eff }}(\rho, z)=0\right)$ on the $(\rho, z)$ plane for the same set of parameters with (a).

In general the effective potential of the MN metric forms various distinct wells in the $(\rho, z)$ polar plane (see Fig. 1b). For $E<1$ there are only bound orbits, since then the potential wells form CZVs that are closed and are not extending to infinity. As explained in [16] for a prolate perturbation of Kerr metric $(q<0)$ for a range of $E, L_{z}$ values there is a distinct outer well and a lobe-like well which is connected to the horizon within which we get plunging orbits. These two wells can be connected for a suitable set of orbital parameters. On the other hand for oblate perturbations of the Kerr metric $(q>0)$ a new well shows up near the central region, in which a new set of bound non-plunging orbits live. Therefore, in the latter case there is a pair of closed CZVs, an inner one and an outer one (Fig. 1), which again can merge for a suitable range of orbital parameters. It should be noted that the outer well is a shallow one that resembles a lot the potential well of the Kerr metric. However the new inner well is a much deeper one with intricate shape (part of this well is shallow but there is also a very deep basin in it). Except from the regions of the non-plunging orbits, in Fig 1 $1 \mathrm{~b}$ the reader can discern 5 more lobe-like regions of permitted motion which extend from the event horizon $(\rho=0)$ to $\rho \lesssim 0.7 M$. These regions contain plunging orbits, a fact that excludes them from the purpose of our paper, which is to study the implications of non-integrability in bound non-plunging orbits.

The lower the energy, the higher is the effective potential and the two distinct CZVs (if they are distinct) move further apart. The effect of $L_{z}$ is the opposite of $E$ : the higher the $L_{z}$, the CZVs move further apart. Finally the 
effect of the source's spin is to bring the two CZVs further apart for lower values of $S / M$. The last conclusion comes mainly from the fact that lower $S / M$ values render the function $\omega$ in the metric less important; hence the effect of the $L_{z}$ term in Eq. (14) effectively grows. The inner well moves closer to the central region and eventually disappears, when the "anomalous quadrupole" $q$ goes to zero from positive values. On the other hand the outer well does not move or change its shape substantially for a wide range of $q$ values.

Furthermore, the distinct inner well (the one that arises for positive $q$ values) is very close to the central region where anomalous regions are located. E.g. the ergoregion can extend into the interior region of the inner well. This case will be examined carefully later on when we analyze the orbits in this region. Fortunately the region of closed time-like curves remains outside the inner well or it merely touches its boundary, at least for the range of parameters which we have analyzed. We want to ensure that the orbits studied do not enter the region of closed-timelike curves; if that happened it would lead us to odd behavior that would have no clear physical meaning.

\section{THE GEODESIC ORBITS IN MN COMPARED TO KERR}

\subsection{The theoretical basis}

In order to present the new observable features that arise in the MN spacetime (as well as in any other similar non-Kerr spacetime) in contrast with the Kerr spacetime, we will describe in brief some basic theorems of dynamical systems that apply in the case of geodesic orbits in the MN spacetime.

Dynamical systems that are described by a Lagrangian function like (8), can be described through a Hamiltonian function as well by simply applying a Legendre transformation on the corresponding Lagrangian. The Hamiltonian function that describes geodesic orbits in a MN metric (as well as in the corresponding Kerr one, if one sets $q=0$ ) is

$$
H=p_{\mu} \dot{x}^{\mu}-L=\frac{1}{2 \mu} g^{\mu \nu} p_{\mu} p_{\nu}
$$

where instead of the velocities $\dot{x}^{\mu}$ (with respect to proper time) we use the corresponding momenta $p_{\mu}=\frac{\partial L}{\partial \dot{x}^{\mu}}=\mu g_{\mu \nu} \dot{x}^{\nu}$ to obtain the final Hamiltonian form.

The specific Hamiltonian that describes geodesic orbits in a MN spacetime, which includes the Kerr spacetime as a special case, has no dependence on the coordinates $t$ and $\phi$, due to stationarity and axisymmetry of the spacetime, respectively. Thus the corresponding momenta $p_{t}$ and $p_{\phi}$ are conserved, that is $p_{t}$ and $p_{\phi}$ are integrals of motion. These conserved momenta are nothing but the quantities $-\mu E$ and $\mu L_{z}$, respectively, that we used earlier. Furthermore, since $d H / d \tau=0$ (autonomous Hamiltonian), $H$ itself is a third integral of motion, namely $H=-\mu$. The first two integrals of motion can be used to reduce the number of degrees of freedom from 4 to 2 ; for example one could use only the coordinates $\rho, z$ to fully describe this 2 -dimensional system. The value of the rest two coordinates $(\phi(\tau), t(\tau))$ along the geodesic orbit could be inferred afterwards by simply integrating Eqs. (11112), as discussed in the previous section. Thus the phase orbits lie on a 3-dimensional hypersurface of the corresponding 4-dimensional phase space, because of the conservation of $H$.

Now, if such a Hamiltonian system of 4 degrees of freedom is an integrable one, that is there is one more integral of motion $I_{4}$ which is independent from the previous ones and is in involution with them $\left(\left\{I_{4}, I_{i}\right\}=0\right.$, where $I_{i}$ represents the first 3 integrals of motion and $\{\cdot, \cdot\}$ represents a Poisson bracket), then the phase orbits lie on 2dimensional surfaces. The Kerr spacetime is an example of such an integrable case with the Carter constant being this new extra integral of motion. This is exactly what makes the Kerr spacetime a very distinct member of the broad family of stationary, axisymmetric and asymptotically flat spacetimes. Moreover the natural formation of Kerr black holes through gravitational collapse of astrophysical compact bodies according to the no-hair theorem, renders the Kerr spacetime a unique highly symmetric case of extreme astrophysical interest that has been extensively studied for a long time [19, 23].

On the other hand any kind of generic perturbation of a Kerr metric that maintains the stationarity and axisymmetry, most probably does not possess a 4-th integral of motion, as in Kerr. This new spacetime could arise from a non-conventional astrophysical process that leads to other types of ultra-compact objects, or it could simply be produced by an axisymmetric distribution of matter around a Kerr black hole (i.e. an accretion disc).

In an integrable autonomous system of 2 degrees of freedom, like the one describing the geodesic orbits in a Kerr metric, the bound orbits lie on 2-dimensional tori in the 4-dimensional phase space. The values of the integrals of motion (the Hamiltonian and the Carter constant) fully characterize these tori; the tori that correspond to different Carter-constant values are nested within each other, while the Hamiltonian value defines the overall scale of the phase space. If one considers a 2-dimensional surface that cuts through a foliage of such tori (called Poincaré surface of section), each torus defines a closed curve on this surface (see e.g. 24]). This curve is called an invariant curve. Each 
torus corresponds to a characteristic pair of frequencies (one for each angle variable). Not only the two frequencies, but also the ratio between them varies continuously from one torus to the next. If the ratio of the frequencies is an irrational number, a phase-orbit continuously winds around its corresponding torus covering densely the surface of the torus. This kind of orbit is called quasiperiodic. A quasiperiodic orbit goes repeatedly through a surface of section defining a succession of points which eventually cover densely the corresponding invariant curve on the surface of section (called quasiperiodic invariant curve). In the special case where the ratio of frequencies is a rational number $n / m(n, m \in \mathbb{N})$ the phase-orbit repeats itself after $m$ windings; then the orbit is periodic and the corresponding torus is called resonant. In this case the resonant invariant curve consists of an infinite number of $m$-multiplets of periodic points. Each $m$-multiplet represents an $m$-multiple periodic orbit.

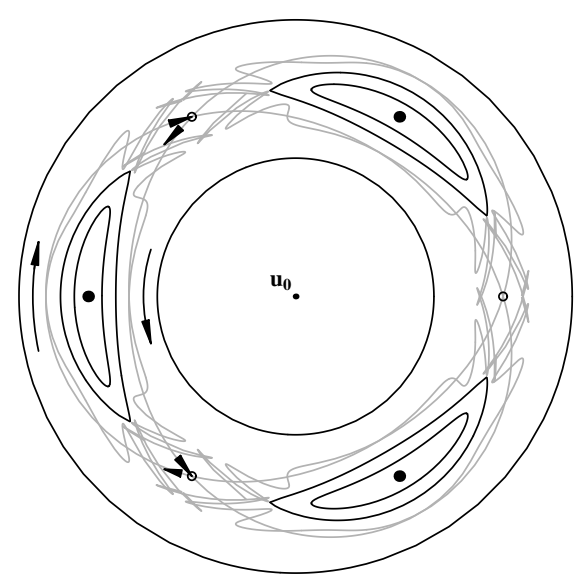

FIG. 2: A schematic representation of a surface of section of a nonintegrable system of 2 degrees of freedom. On the surface there are depicted two KAM curves; one outside and one inside the chain of islands. The particular chain of islands in between consists of three islands ( $m=3, n=1)$, each one arising around each of the three stable points (shown as filled circles). Each island consists of a whole set of KAM curves nested within each other. Between the successive islands there are three unstable points (shown as open circles) from which the asymptotic curves (gray curves) emanate surrounding the islands by a thin chaotic layer (here magnified). The arrows indicate the flow around the leftmost island. The $\mathbf{u}_{0}$ indicates the central fixed point around which the KAM curves and the Birkhoff islands are formed.

According to the Kolmogorov-Arnold-Moser (KAM) theorem [20], if any integrable system gets perturbed (without altering its dimensionality) most of its tori are deformed but they are not destroyed. These tori are called KAM tori. Thus the corresponding surface of section looks very much like the surface of section of the corresponding integrable system. The quasiperiodic invariant curves in nonintegrable systems are called KAM curves. This is exactly the behavior exhibited by most of the geodesics in the MN spacetime, at least for bound orbits in the outer allowed region (see [16]). However, there are qualitatively new characteristics that are related with the resonant tori of a perturbed integrable system. According to the Poincaré-Birkhoff theorem [25], when a system deviates slightly from an integrable one, the resonant invariant curve disintegrate and only a finite even number $\left(2 k m\right.$, where $\left.k \in \mathbb{N}^{*}\right)$ of the periodic points of period $m$ survive. This means that from the resonant torus only $2 k$ periodic orbits survive. Half of the surviving periodic points are stable while the rest are unstable. If we imagine a closed curve which passes from all the surviving points of the disintegrated resonant curve, then the stable and the unstable periodic points interchange along that closed curve forming the Birkhoff chain. Around each stable periodic point there is a set of nested KAM curves forming an island of stability (see Fig. (2). A phase-orbit of such a resonant case visits all the $m$ islands of the $n / m$-resonance, moving successively to the next $n$-th island along the imaginary aforementioned closed curve at every winding, forming eventually the KAM curves inside every island. The interesting feature of these islands, that we exploit in our paper, is that every regular (non-chaotic) orbit that belongs to a chain of islands is characterized by the same rational ratio of frequencies (characteristic of the particular chain of islands), no matter on which KAM curve inside the chain of islands it belongs. This property is not shared by the non-resonant KAM curves, since the ratio of frequencies on them is irrational and it varies smoothly from one KAM curve to another.

Finally, there is a region around the Birkhoff chain of islands that consists of chaotic orbits. These chaotic orbits appear mainly in the neighborhood of the unstable points of the Birkhoff chain (see Fig. 2). The chaotic orbits arise from the asymptotic curves of the unstable periodic orbits, which are forced to follow a very complicated pattern of multiple intersections when they approach each other. The intersections of the asymptotic curves are called homoclinic points and the corresponding orbits are called homoclinic orbits. The chaos produced by the homoclinic orbits is called homoclinic chaos. As long as the deviation from the integrable case is small the chaotic areas on a 
surface of section are restrained on a very thin ring, surrounding the Birkhoff islands of stability, which is thicker near the unstable points. For higher deviations from an integrable case the KAM curves intervening between two different resonances are destroyed. Then two Birkhoff chains which were initially isolated by intermediate KAM curves can communicate (resonance overlap) 26 28], i.e. the asymptotic curves that emanate from unstable points of different chains intersect each other at the so-called heteroclinic points, forming heteroclinic orbits. These orbits produce the so-called heteroclinic chaos. The chaotic behavior in a resonance overlap is stronger than in the homoclinic one.

It should be emphasized that the $q$-parameter that characterizes the deviation of the MN metric from the corresponding Kerr metric is not an absolute measure of the deviation of the corresponding geodesics, since different regions of the MN spacetime have more or less deformed metric components (with respect to their Kerr values). For example the greater the distance from the central point of the field, the smaller is the effect of the perturbed moments of the spacetime, since there the spacetime is mainly characterized by its lowest few moments; there the higher multiple moments are almost unimportant for the shape of the geodesic orbits. Therefore one anticipates that the system of MN geodesic orbits should be more organized in its outer region, in contrast with its more chaotic inner region.

\subsection{Rotation number}

When the perturbation parameter of a 2-dimensional nonintegrable system is sufficiently small, the Birkhoff islands of stability are very thin and their detection on a surface of section is quite cumbersome; fine tuning is needed to find suitable initial conditions of orbits that develop into a chain of islands on a surface of section. However, these islands can be detected by a more sophisticated technique. The islands of stability lie around a resonant periodic orbit which is characterized by a commensurate ratio of frequencies $\nu=\omega_{1} / \omega_{2}=n / m$, where $\omega_{1}, \omega_{2}$ are the fundamental frequencies corresponding to the two angle variables while $n, m$ are integers. This ratio characterizes not only the resonant periodic orbit of the island (represented by a set of $m$ stable points on a surface of section), but all the KAM orbits around them belonging to the particular chain of islands. Although each distinct KAM orbit in an island is characterized by a different pair of frequencies $\omega_{1}, \omega_{2}$, all such KAM orbits are marked by the same commensurate ratio $\nu$ with the central periodic orbit.

An index that has been used for detecting chaos in classical nonintegrable systems of two degrees of freedom is the rotation number. This index computes the ratio of the fundamental frequencies $\omega_{1}, \omega_{2}$ and therefore could be used to detect the islands of stability as well [28 30]. In order to evaluate the rotation number we first identify the fixed point $\mathbf{u}_{0}$, around which the KAM curves (not the ones a Birkhoff island consists of) are formed (Fig. 2) creating a formation known as the main island of stability. In our case $\mathbf{u}_{0}$ is the point that corresponds to the periodic orbit which crosses the equatorial plane at only one point with $\dot{\rho}=0$ moving towards the positive part of the $z$-axis (see Fig. 3a). It should be noted though that this orbit is periodic with respect to its projection on the $(\rho, z)$ plane, but if one considers the $\phi$ coordinate of the orbit as well, the orbit is not necessarily periodic then. Now we define the position vector of the $i$-th crossing point $\mathbf{u}_{i}$ of a phase orbit on a surface of section to be

$$
\mathbf{r}_{i}=\mathbf{u}_{i}-\mathbf{u}_{0}
$$

that is its position with respect to $\mathbf{u}_{0}$. Next we compute the angles $\theta_{i} \equiv$ angle $\left(\mathbf{r}_{i+1}, \mathbf{r}_{i}\right)$ between two successive position vectors, the so-called rotation angles, and finally we calculate their mean value for a large number (theoretically an infinite number) of crossings, divided by $2 \pi$. This number provides the so-called rotation number $\nu_{\theta}$, i.e.

$$
\nu_{\theta}=\lim _{N \rightarrow \infty} \frac{1}{2 \pi N} \sum_{i=1}^{N} \theta_{i}
$$

and it measures the average fraction of a circle by which successive crossings advance.

The rotation number usually appears to grow monotonically as long as we cross KAM curves that surround the central fixed point $\mathbf{u}_{0}$. The strict monotonicity is interrupted by KAM curves belonging to resonant islands of Birkhoff chains surrounding the fixed point $\mathbf{u}_{0}$. Within a resonant island the rotation number remains fixed at a constant rational value. This rational number, as already stated, is characteristic of that island of stability, since it is the outcome of the resonance between $\omega_{1}, \omega_{2}$. It should be also noted that in a chaotic region of a nonintegrable system the rotation number fluctuates irregularly from point to point, so its behavior appears to be smooth only in the region of regular orbits. For a slightly perturbed integrable system the chaotic layers around the Birkhoff chains of islands are so thin that the fluctuating behavior of the rotation number is hardly observable.

The rotation number is not just a "mathematical" tool, it is actually an "observable" quantity. The frequencies $\omega_{1}$, $\omega_{2}$ can be derived from the signal of the gravitational waves, through a Fourier analysis. By monitoring the evolution of the frequencies encoded in a gravitational wave signal coming from an EMRI, we monitor the rotation number and when we observe stationarity of its value as time progresses we can infer that the corresponding EMRI is evolving in a non-Kerr spacetime. 


\subsection{The outer region}
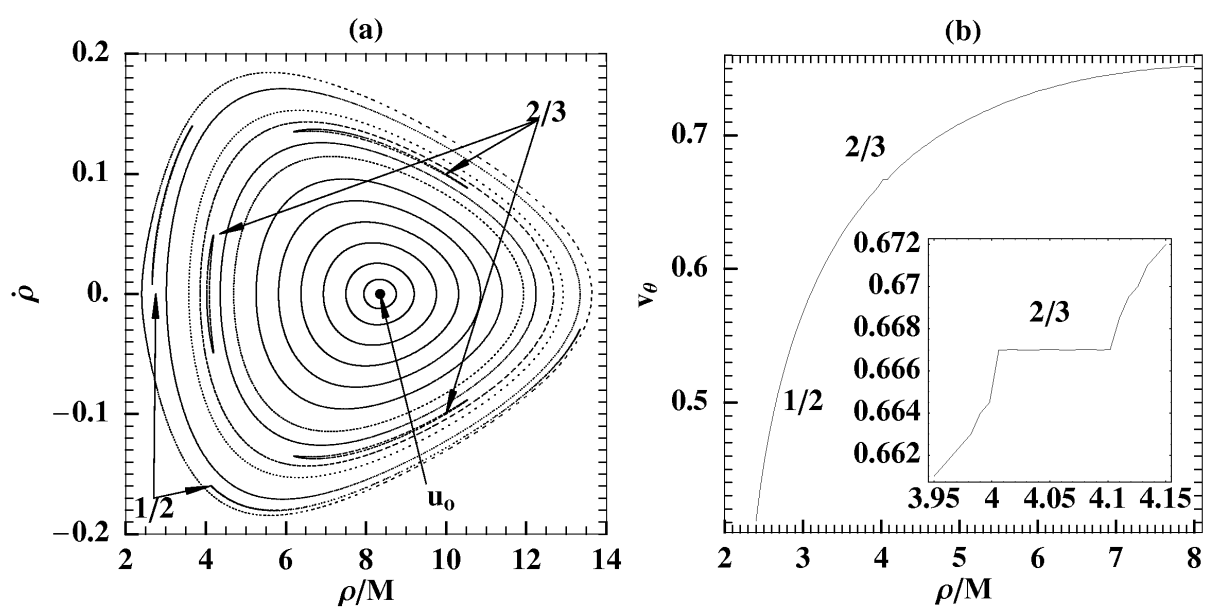

FIG. 3: (a) The surface of section of the outer region on the $(\rho, \dot{\rho})$ plane for the parameter set $E=0.95, L_{z}=3 M, \chi=$ $0.9, q=0.95 . \mathbf{u}_{0}$ indicates the fixed point at the center of the main island. (b) The rotation number vs. $\rho$ along the line $\dot{\rho}=0$ of the surface of section presented in (a). Embedded in (b) is a detail of the rotation curve around the 2/3-resonance.

As it was demonstrated in Fig. 1, which was characterized by a particular set of parameters of the MN spacetime and of the test particle, there may be two separate regions where bound geodesic orbits are allowed to develop, namely the inner region (closer to the central singularity) and the outer region (farther from the central singularity). In this section we explore the dynamical properties of the outer region by studying the surface of section $z=0(\dot{z}>0)$ on the $\rho, \dot{\rho}$ plane shown in Fig. 3a. By inspection this surface of section seems to be filled densely with KAM curves, and only two thin chains of islands of stability are discerned; no visible sign of stochasticity is present. However, the existence of these two Birkhoff chains, the one of multiplicity 2 (labeled as 1/2) and the other of multiplicity 3 (labeled as 2/3), implies that the system is actually nonintegrable and therefore chaos should be present [27, 28]. In fact the surface of section should be densely filled with other Birkhoff chains of islands as well, but their detection demands a very detailed scan of the surface of section. Even the revealing of the two chains of islands which we found would be a very hard task, if we hadn't employed the rotation number in order to explore the fine details of the phase space that did not show up in the coarse sweep of initial conditions performed by Gair et al [16].

As we have already explained the rotation number is an appropriate tool to detect the islands of stability, since all the regular orbits belonging to an island share a common characteristic rational value of the rotation number. The implementation of the rotation number in our case is presented in Fig. 3 $\mathrm{b}$. The scan begins from the central point $\mathbf{u}_{0}$ and goes inwards, towards $\rho=0$, along the line $\dot{\rho}=0$ on the surface of section seen in Fig. 3 $\mathrm{k}$. The produced rotation curve is a decreasing function of the distance from the center $\mathbf{u}_{0}$ of the main island of the outer region. The curve appears to be strictly monotonic except from a narrow plateau, a constant value of the rotation number within a $\rho$-interval, lying near $\rho=4$ (see the magnified plot of the embedded figure in Fig. $3 \mathrm{~b}$ ). This plateau is labeled by the value of the corresponding rotation number $\nu_{\theta}=2 / 3$, and is related to the period-3 chain of islands of Fig. 3 $\mathrm{k}$.

However no plateau appears in Fig. 3b for the period-2 island of stability $(1 / 2$-resonance), even though the rotation curve passes through the value $\nu_{\theta}=1 / 2$. This can only mean that the island is very thin along the line $\dot{\rho}=0$ and our step along the scanning line is not sufficiently small to discern a corresponding plateau. Instead by moving along the line $\dot{\rho}=0.05$, where the particular period-2 island is thicker, we find a discernible plateau (Fig. (4).

In the integrable Kerr case no such plateaus are expected to exist. According to the analysis done in [31] for the bounded geodesic orbits in the Kerr spacetime, the Hamilton function expressed in action angle variables does not depend explicity on the angle variables and therefore the integrable Kerr spacetime has no resonant islands. Thus the rotation curve in a Kerr case is a strictly monotonic function of the distance from the center; the corresponding resonances being presented simply by a set of periodic points that lie along a single curve on a surface of section instead of a chain of islands with finite thickness. This difference, along with the analysis of inspiraling orbits presented in section 4 is an effect that can be quantitatively checked by the gravitational wave detectors.

Once again we note that other resonances corresponding to any rational value of $\nu_{\theta}$, that are not depicted in Fig. 3 , are so narrow that they are really very difficult to be pinpointed on a surface of section.

As mentioned before the finite width of the resonant islands in a non-Kerr metric, in contrast to the zero-width 


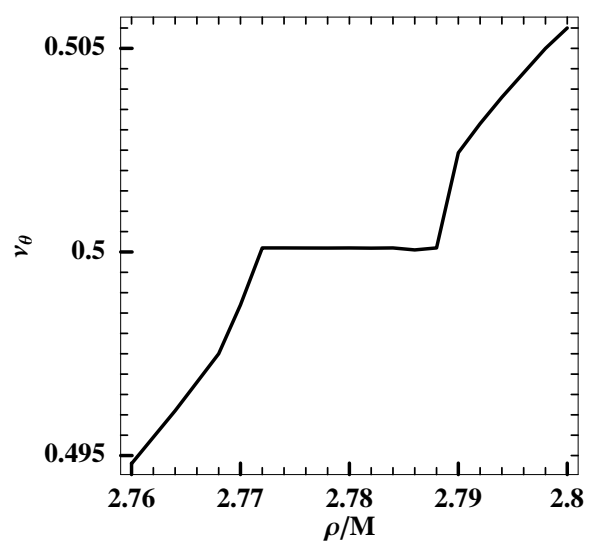

FIG. 4: The rotation curve along the line $\dot{\rho}=0.05$ which is crossing the $1 / 2$-resonance on the surface of section of Fig. 3 a.

of the corresponding resonances of the Kerr metric is the new feature that we propose to exploit. Thus the size and the position of the islands are of great importance in our analysis. The size and the position of the resonant islands depend on both the physical parameters of the MN metric $(q$ and $\chi)$ and the parameters of the orbit itself $(E$ and $\left.L_{z}\right)$. For example if we keep the three parameters $E, \chi, q$ fixed and plot the width $w$ of the period-3 island along the $\dot{\rho}=0$ line on the surface of section $z=0$ as a function of the angular momentum $L_{z}$, we get Fig. 5 a. On the other hand in Fig. 6 a we plot along the same line, for the same set of parameters, the positions $\rho_{c}$ of the central point $\mathbf{u}_{0}$ of the main island (diamonds), the center of the multiplicity-3 leftmost island (black squares), the outer and inner boundary of the outer region (open triangles and open circles respectively), the inner and outer boundary of the inner region (black triangles and black circles respectively). The width $w$ of the island increases as we increase $L_{z}$, till it reaches its maximum width when $L_{z} \approx 2 M$ (Fig. 5 ). Then it starts becoming thinner again. This behavior is due to the fact that as we increase $L_{z}$ the potential well becomes more compressed along the equator. Therefore as the particular island moves away from the central point $\mathbf{u}_{0}$ it is compressed by the boundary of the well (high $L_{z}$ values), while if the island moves close to the center, it is finally compressed in the region around the central point $\mathbf{u}_{0}$ (low $L_{z}$ values) (cf. Figs. $5 \mathrm{a}, 6 \mathrm{a}$ ).

If instead of varying the angular momentum we vary the energy $E$ and measure the width $w$ and the positions $\rho_{c}$, we obtain Figs. 5b and 6b respectively. Again as we increase the energy the allowed region is expanded and thus the thickness of the island increases as well. On the other hand there is a lower energy threshold below which the island of the 2/3-resonance is compressed to zero thickness due to shrinkage of the whole CZV.

If we keep the energy and the angular momentum at their initial value $E=0.95, L_{z}=3 M$ and vary the quadrupole deviation $q$, the width $w$ of the island and the positions $\rho_{c}$ change as shown in Figs. 5 ; and 6respectively. The $q$ parameter is actually the parameter that controls the non-integrability of the system since for $q=0$ it describes a Kerr metric which is integrable. Thus it is anticipated that as $q$ increases the width increases as well. Moreover the displacement of the 2/3-resonance with respect to the boundaries of the CZV works in favor of the island's expansion as long as the island is away from the main center $\mathbf{u}_{0}$. For example, as is shown in Fig. 55 for $q$ below $\simeq 0.4$ the thickness has been shrunk to almost zero, since the island has moved close to the boundaries of the CZV (Fig. 6 $\mathrm{c}$ ). It should be noted that this limiting value of $q$ has no universal character, but corresponds to the specific values of the other parameters.

Finally the role of the spin parameter $\chi$ of the metric is qualitatively similar to that of the angular momentum of the orbit. The island moves away from the central point $\mathbf{u}_{0}$ as $\chi$ increases (Fig. 6 d), while the thickness of the island increases as well (Fig. 5 $\mathrm{d}$ ). At least in the case we have studied (corresponding to the specific values for the other parameters) when $\chi \simeq 1$ the island has not yet come very close to the boundaries of allowed orbits (Fig. 6f); thus the island has not been forced to shrink (Fig. [5 d).

For all the above range of parameters the period-3 island exists along with other islands of stability from different Birkhoff chains. In general when the Kerr spacetime is even slightly perturbed and the Carter constant is destroyed (ceases to be a constant), the Birkhoff chains with their islands of stability show up. The general behavior of the most prominent islands of resonance in a slightly perturbed Kerr metric are expected to exhibit similar behavior with the 2/3-island for different ranges of the parameters $E, L_{z}, q, \chi$ of the $\mathrm{MN}$ metric, since the parameters $E, L_{z}$ of the orbit, and $\chi$ of the metric play a similar role for any kind of orbit in a generically perturbed Kerr metric. The $q$ parameter of the MN may be replaced by another set of one or more parameters in a generic perturbed Kerr metric that will control the deviation of the metric from the Kerr metric. 
(a)

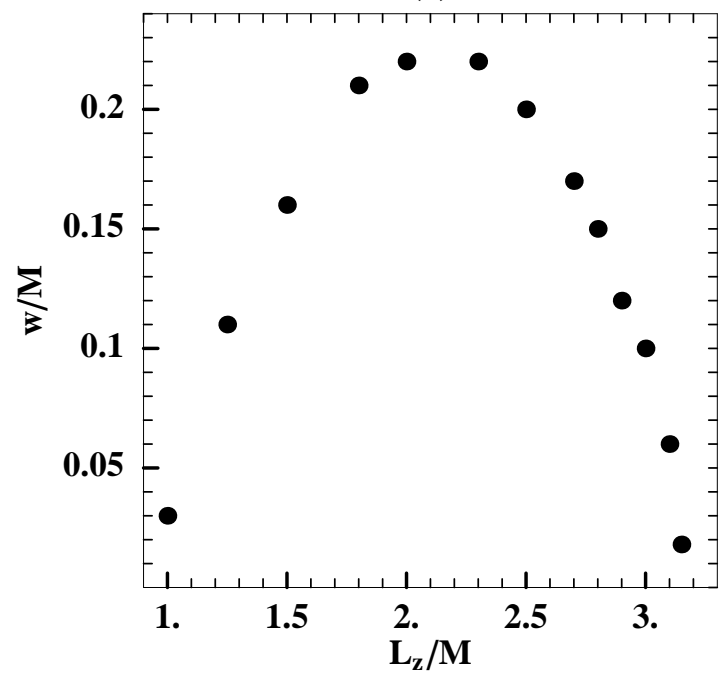

(c)

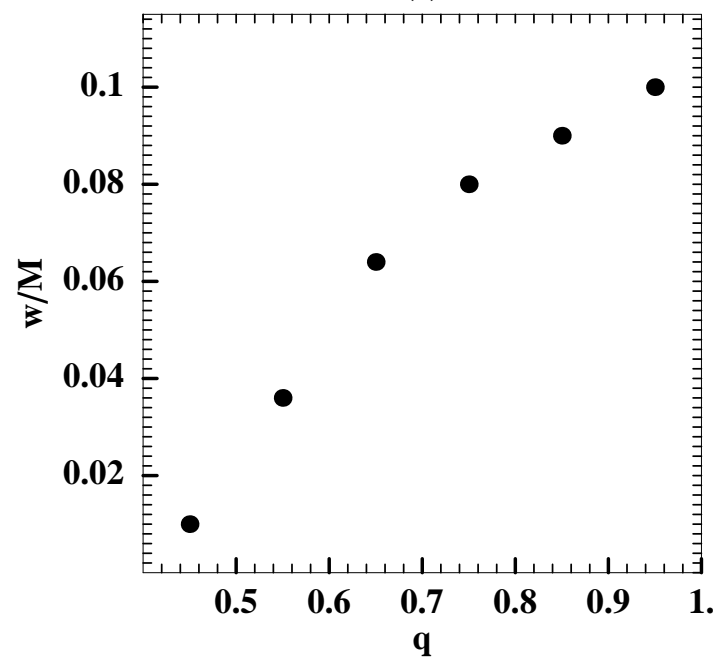

(b)

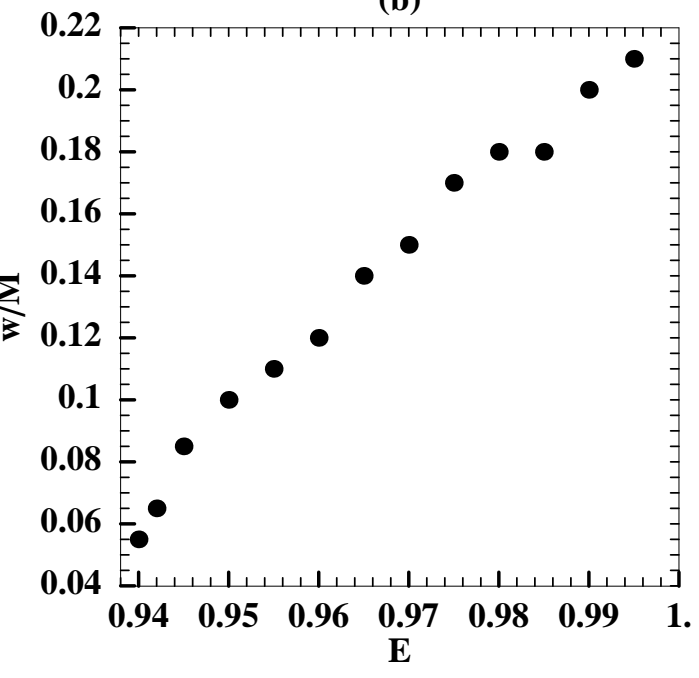

(d)

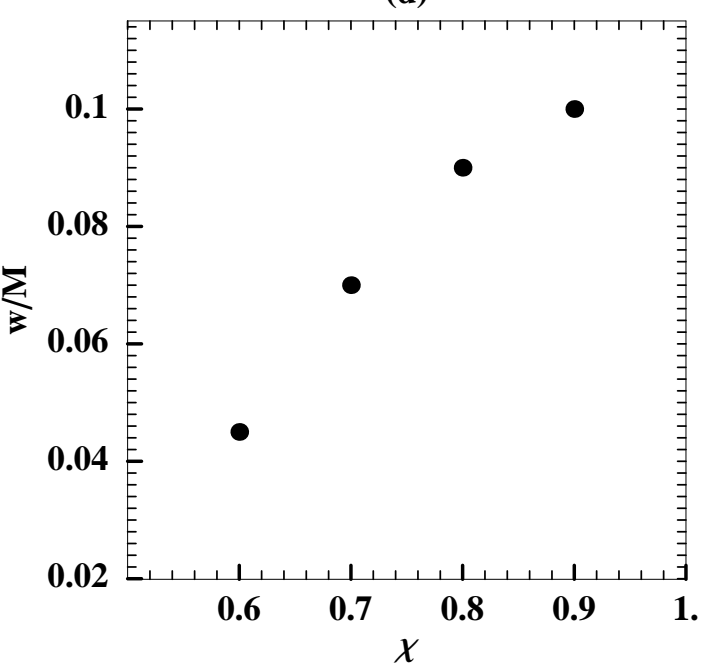

FIG. 5: The width $w$ of the period-3 leftmost island of stability along the line $\dot{\rho}=0$ (the thickest region) for different sets of the $E, L_{z}, q$, and $\chi$ parameters. (a) The $w$ vs. the angular momentum $L_{z}$ when $E=0.95, q=0.95, \chi=0.9$. (b) The $w$ vs. the energy $E$ when $L_{z}=3 M, q=0.95, \chi=0.9$. (c) The $w$ vs. the quadrupole deviation $q$ when $L_{z}=3 M, E=0.95, \chi=0.9$. (d) The $w$ vs. the spin parameter $\chi$ when $L_{z}=3 M, E=0.95, q=0.95$. Apparent non-smooth behavior of the plots is an artifact of the accuracy used to measure the width.

In order to numerically integrate the orbits and produce Figs. 35 , we have applied a sixth order Runge Kutta integration scheme with a constant step of integration $\delta s=10^{-1}$, except for the cases in which an orbit reaches the surface of section; then the integration step was gradually reduced to $\delta s=10^{-8}$ in order to get a fine approximation of the surface of section at $z=0$. To ensure that the numerical errors do not affect the evolution of the orbits and our conclusions with regard to their characteristics, at every step we evaluate the Lagrangian per unit test mass $L_{n} / \mu$ and compare it to its expected value $L / \mu=-1 / 2$. The relative error of the integration $\Delta L / L=\left|\left(L_{n}-L\right) / L\right|$ after $10^{3}$ crossings through the $z=0$ surface of section did not exceeded the order of $\Delta L / L=10^{-10}$. In order to get a smaller relative error we tried to use an even smaller step of integration for these orbits. This was time consuming though, without improving substantially any quantitative information we gained with a coarser integration step; therefore we decided to keep the initial $\delta s=10^{-1}$ step. 
(a)

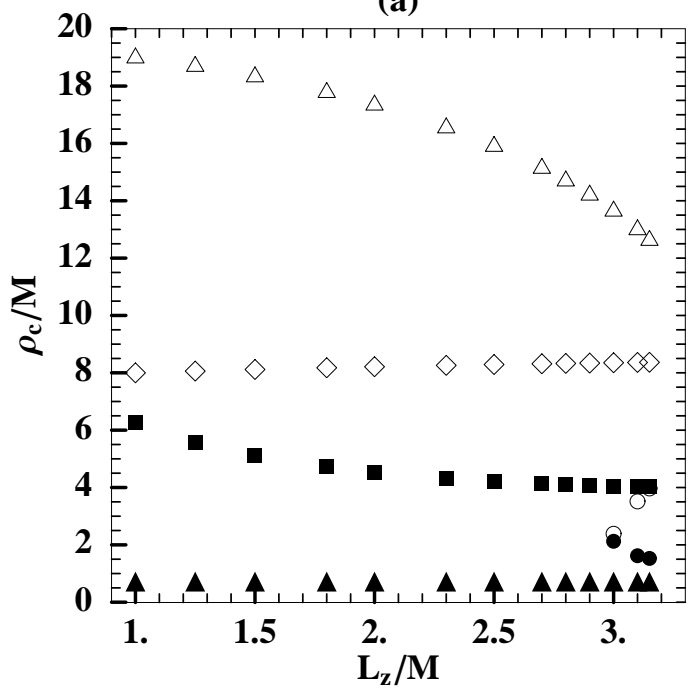

(c)

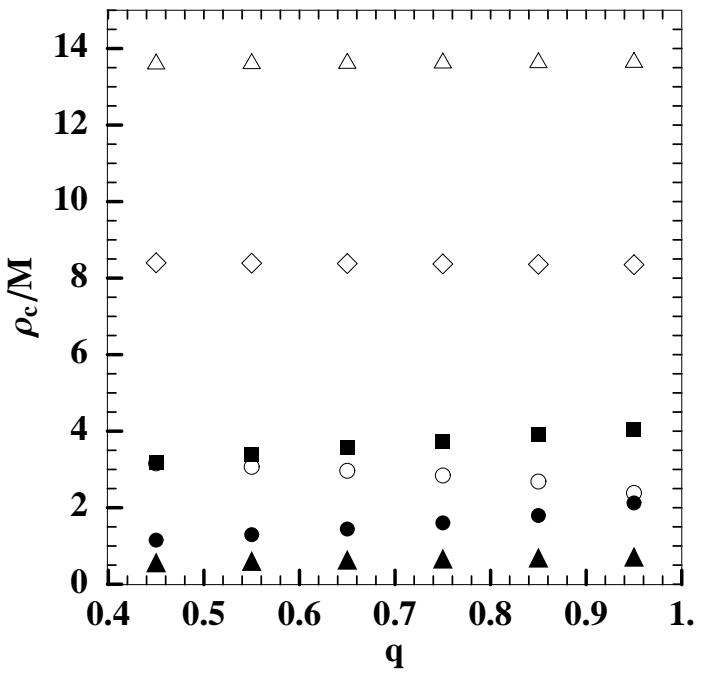

(b)

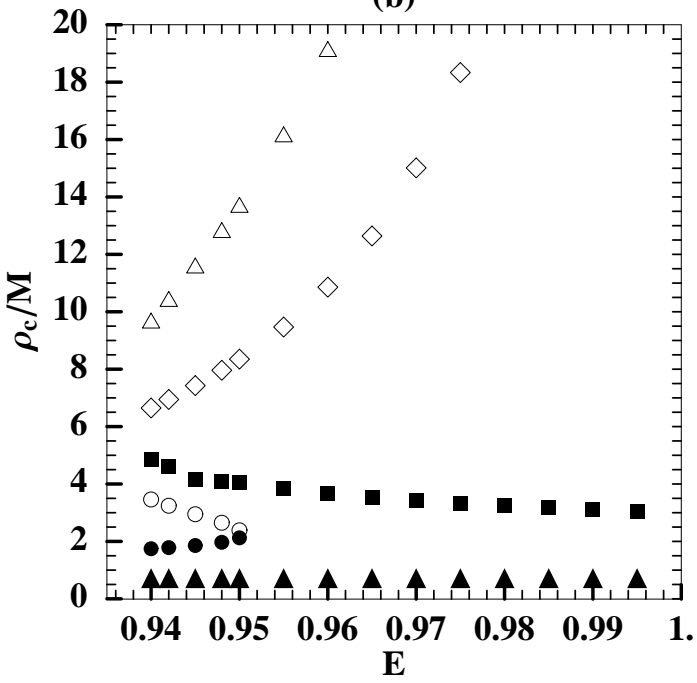

(d)

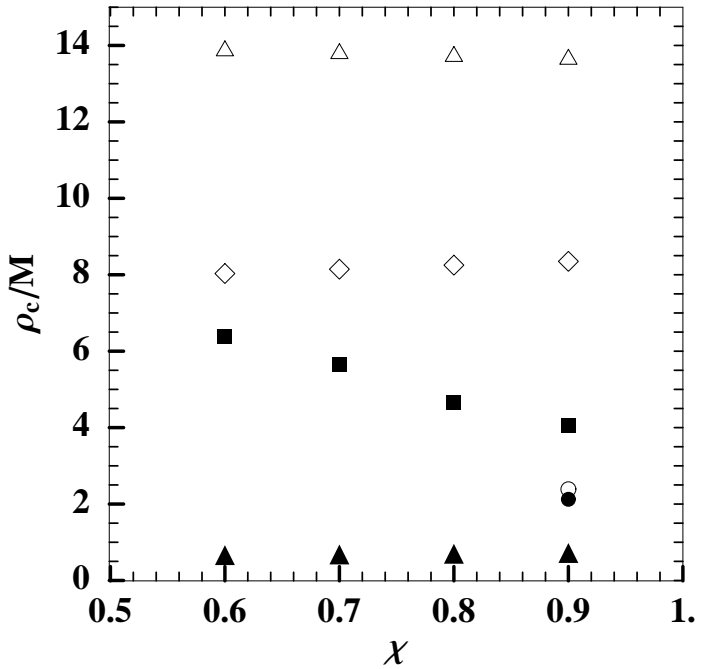

FIG. 6: The radial positions $\rho_{c}$ of 6 different locations of interest along the line $\dot{\rho}=0$ on the surface of section $z=0$ for the same sets of parameters $E, L_{z}, q, \chi$ as in Fig. 5. The diamonds represent the central point $\mathbf{u}_{\mathbf{0}}$ of the main island, the black squares represent the center of the multiplicity-3 leftmost island, the open triangles and open circles represent the outer and inner boundary of the outer region respectively, while the black triangles and black circles represent the inner and outer boundary of the inner region respectively. When the open and the black circles merge the outer and the inner regions get connected through a neck.

\subsection{The inner region}

In contrast to the outer region, which contains mainly regular orbits, the inner region displayed in Fig. 7 has a more complicated structure. On the left side of Fig. $7 \mathrm{~h}$ there is a period-1 island of stability. Around the center of the same figure there are also three islands of stability belonging to a period-3 chain (these islands are depicted in detail in Fig. (7b). The rest of the inner region seems to be occupied by chaotic orbits, in accordance with the findings of Gair et al [16]. A domain of the surface of section that is mainly occupied by chaotic orbits surrounding a main island of stability, like the region of Fig. $7 \mathrm{k}$ which is filled with scattered dots, is often described as a chaotic sea in nonlinear dynamics literature (see e.g. [28]).

To produce Fig. 7 we applied again a sixth order Runge Kutta integration scheme but now we used an adaptive step of integration which varied from $\delta s=10^{-4}$ to $\delta s=10^{-8}$ in a way that the relative error did not exceeded $\Delta L / L=10^{-8}$. In order to avoid problems with the static limit, which is extended inside the inner region, we 
(a)

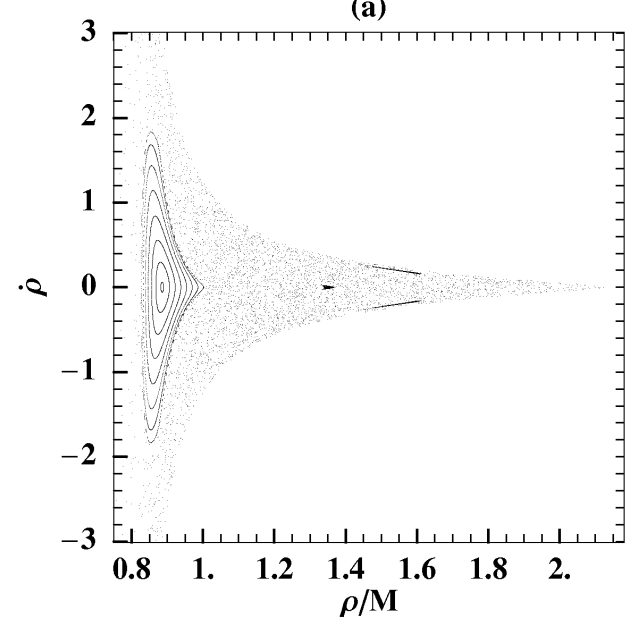

(b)

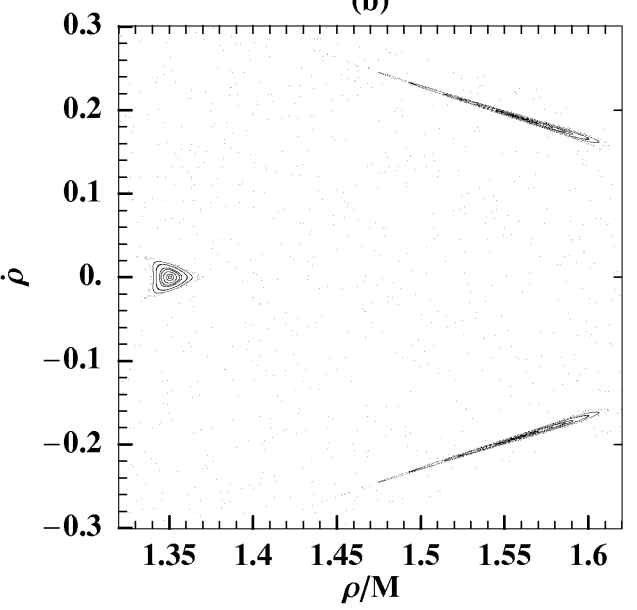

FIG. 7: (a) The surface of section of the inner region on the $\rho, \dot{\rho}$ plane for the parameter set $E=0.95, L_{z}=3 M, \chi=$ $0.9, q=0.95$. (b) A detail of (a) showing islands of stability surrounding a period-3 orbit.

analytically eliminated the zeroes of function $A$ (Eq. (5d $)$ from all the metric components $g_{\mu \nu}$ for which $A$ appears in the denominator, like $g_{\phi \phi}$.

Oddly enough Fig. 7 $\mathrm{h}$ seems not to be in total agreement with Fig. 7 of [16]. The region which in our Fig. 7 $\mathrm{k}$ is occupied by a main island of stability, in Fig. 7 of [16] seems to be visited by a chaotic orbit coming from the chaotic sea. In fact, in a system of 2 degrees of freedom chaotic orbits surrounding an island of stability cannot enter the island itself, because the KAM curves of the island in such systems block such entrances.

The qualitative separation of the inner region in one domain dominated by organized orbits and another one dominated by chaotic orbits could be easily justified by the shape of the corresponding potential well. As shown in the left part of Fig. 1a the potential well of the inner region in which the orbits develop consists of a shallow part (right side of the left well) and a much deeper part located at the innermost part of the inner region (left side of the left well). The chaotic orbits (Fig. 8 a) are the ones that start from the shallow part of the well (see the embedded diagram in Fig. 8b) and eventually enter the deep hole (Fig. 8 8 ) which works as a stochastic scatterer of the orbit. In Fig. $8 \mathrm{~b}$ we see only the upper part of the hole which in full scale reaches the value of $-10^{5}$ for the chaotic orbit seen in Fig. 8 a. Chaos is generated by the abrupt changes of the values of the $V_{\text {eff }}$ along an orbit. On the other hand the organized orbits, which belong to the main island of the inner region, (Fig. 85) move on the periphery of the deep well (Fig. 8 8 ). Finally, the orbits which belong to the chain of islands of the resonance $2 / 3$ (Fig. 86 ) are orbits with well tuned initial conditions in the phase space that start from the shallow region, eventually enter slightly in the deep region but again move mostly on the periphery of the hole itself (Fig. 8 ), thus maintaining their organized character.

\subsection{The neck}

Previously we studied a case in which the outer and the inner regions are separated. Let us see now what happens when the two regions communicate with each other. In order to attain this we have slightly reduced the angular momentum to the value $L_{z}=2.995 M$ without altering the other parameters. Then, as shown in Fig. 9 a short neck that connects the two regions appears at $\rho \approx 2.25 M$. Through this neck, orbits are allowed to move from the outer to the inner region and vice versa.

Although the main characteristics of the two regions have not changed, the "neck" has allowed the formation of heteroclinic sections of the asymptotic curves emerging from unstable periodic orbits lying in the two initially separate regions. Such sections lead to stronger chaos. In our case chaotic orbits that were initially confined between the boundaries of the outer region and the last KAM of the corresponding main island are able to explore the phase space of both regions by escaping through the neck to the more chaotic inner region and returning again through the neck to the thin chaotic layer near the periphery of the outer region. The chaotic layer becomes more prominent when the inner and the outer regions come closer to each other. There is also a well-known phenomenon in nonlinear dynamics called "stickiness" first reported in [32] (for a review see [28]), which could explain the "strange" behavior reported in [16]. The stickiness concerns chaotic orbits which for various reasons stick for a long time interval in a region, close to an invariant curve, so that their behavior on a surface of section may resemble that of regular orbits, 

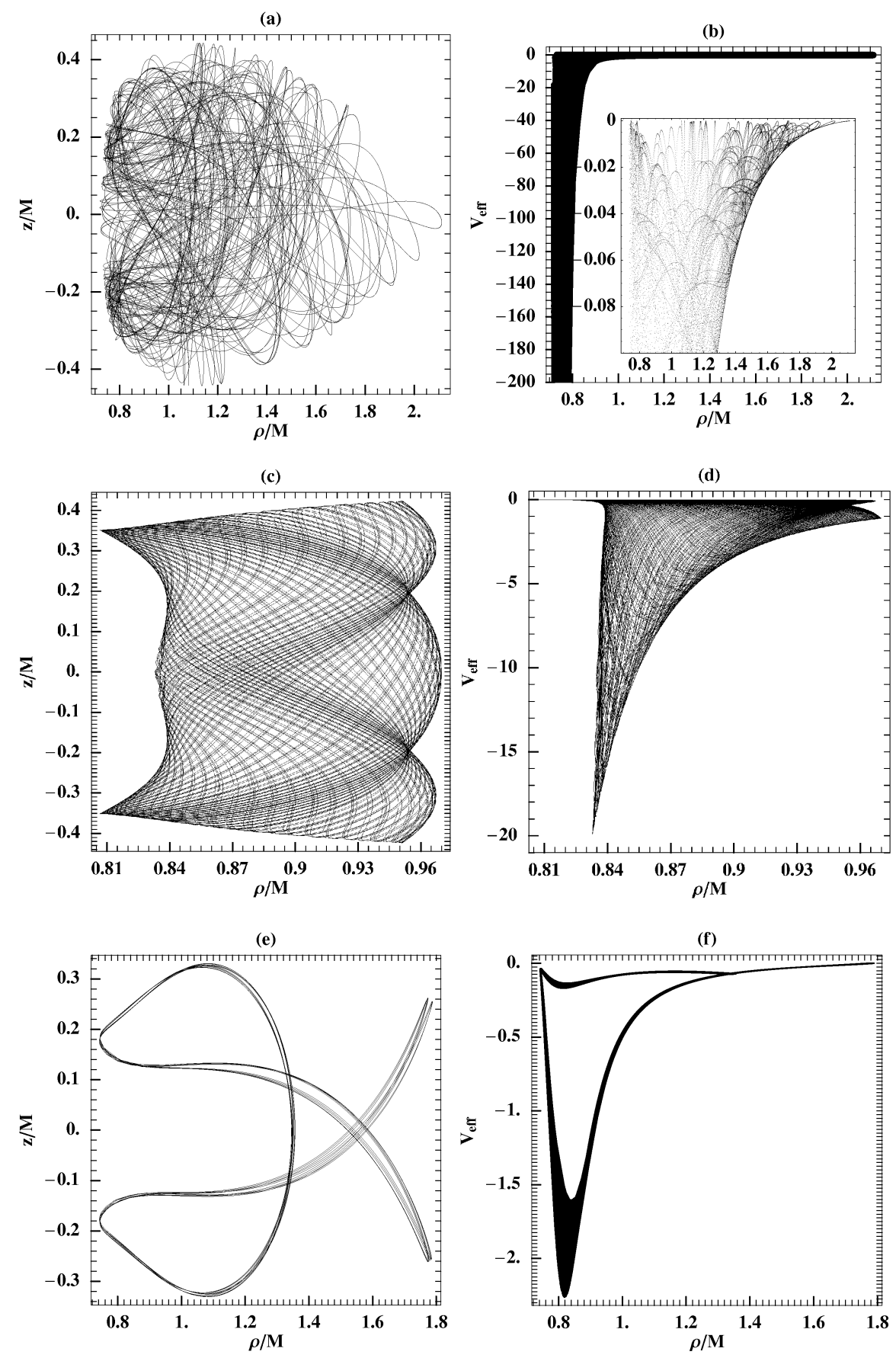

FIG. 8: In the left column various bound orbits of the inner region (Fig. 7) are shown as projections on the ( $\rho, z)$ plane. The projected orbits are: (a) a chaotic orbit, (c) a regular orbit of the main island, (e) a regular orbit of the 2/3-resonance. The right column shows the corresponding evolution of the value of $V_{\text {eff }}$ along the orbit as a function of the instantaneous $\rho$ coordinate. The embedded figure in (b) shows a detail near the upper edge of $V_{\text {eff }}\left(V_{\text {eff }} \simeq 0\right)$.

before extending further away.

The situation described in the previous paragraph is depicted in Fig. 10 which is a $z=0$ surface of section of the region of $\rho$ near the neck. The main island of the outer region is surrounded by a chaotic layer which communicates with the chaotic sea of the inner region. In this "outer" chaotic layer many high-multiplicity islands of stability are present. The borders of this chaotic layer with the region of regular orbits is quite densely populated by chaotic orbits (Fig. 10a). In fact the chaotic orbits near these borders remain close to the regular orbits for a long time before 


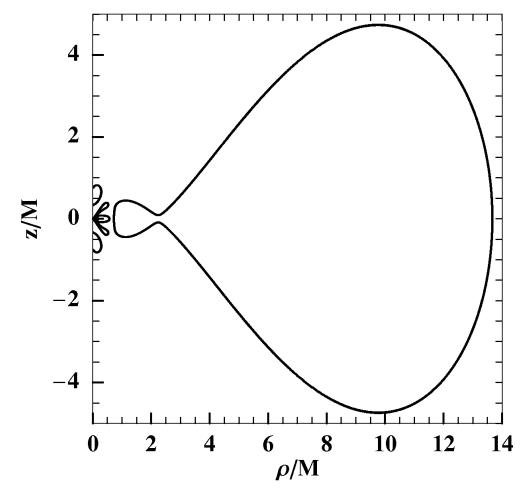

FIG. 9: The CZV for parameter values $E=0.95, L_{z}=2.995 M, \chi=0.9, q=0.95$, where the outer and the inner region are connected through a short neck.

(a)

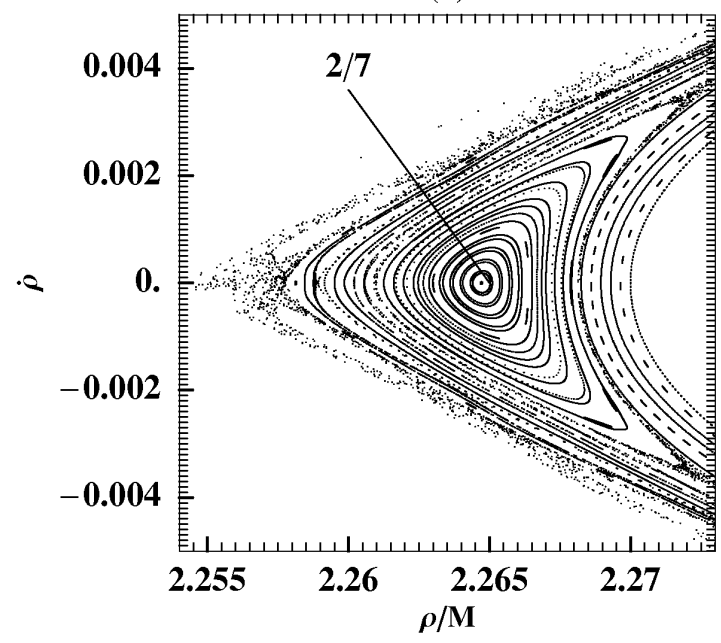

(b)

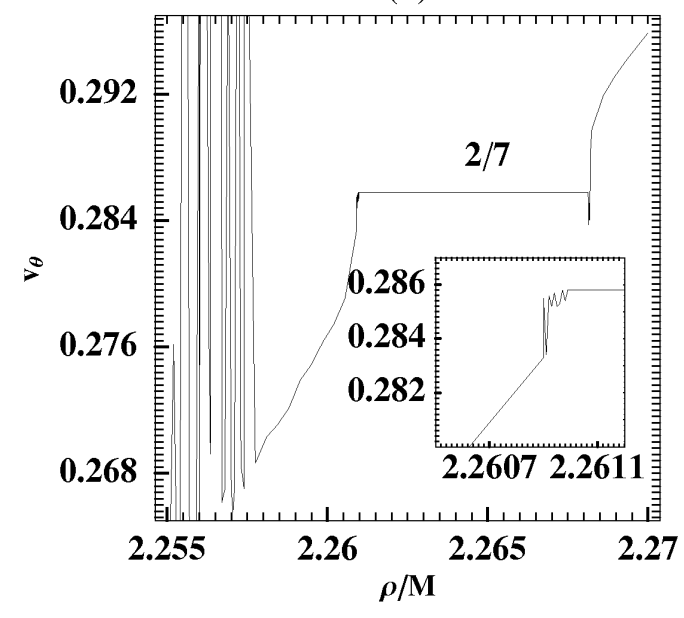

FIG. 10: (a) A detail of the surface of section in the neighborhood of the neck which joins the inner and the outer regions. (b) The rotation curve along the $\dot{\rho}=0$ line of the surface of section presented in (a). Embedded in (b) is the irregular variation of the rotation number just outside the left side of the $2 / 7$-plateau. This irregular behavior is due to the chaotic layer surrounding the corresponding island.

escaping to the main chaotic domain. The sticky zones are near these borders. In order to further explore the area of the outer region near the neck we computed the rotation number along the $\dot{\rho}=0$ line (Fig. 10 $\mathrm{b}$ ). On the left side of Fig. 10b the irregular variations of the rotation number confirm the chaoticity of the orbits that surround the main island of the outer region. On the right of these irregular variations the rotation curve seems to be strictly monotonic, until we reach the resonant island $2 / 7$ (the plateau in the middle of Fig. 10 b). Around the resonant island 2/7 a thin chaotic layer can be discerned on the surface of section, mainly around two unstable points near $\rho \simeq 2.275 M$, $\dot{\rho} \simeq \pm 0.0035$ in Fig. 10 . The chaotic nature of the orbits in that layer produces again an irregular variation of the rotation number (embedded diagram in Fig. 10b). On the right of the $2 / 7$ resonant island the rotation number seems to grow like a strictly monotonic function again.

The fact that chaotic orbits belonging to thin chaotic layers, like the ones described above, tend to stick near regular orbits has a side-effect on their frequency spectrum. As long as the orbits stick near a regular orbit they tend to get two main frequencies like the frequencies corresponding to the regular orbits. When they move away from this regular orbit they lose these two main frequencies and the chaotic noise in their frequency spectrum prevails. However, the orbits can approach again a regular orbit, even the one they departed from, stick around it again for a certain interval of time and exhibit again two main frequencies. If these regular orbits belong to resonances the ratio of the two fundamental frequencies will be for some time a rational number, otherwise it will be irrational. The 
appearance and disappearance of the two main frequencies characterizes the existence of sticky chaotic orbits, thus it signals the existence of non-Kerr spacetime, although this would be a difficult observational task due to instrumental noise in gravitational wave signals. Therefore, we mainly focus our observational method on the regular orbits which correspond to resonances.

\section{INSPIRALING ORBITS}

According to general relativity an object of mass $\mu$ orbiting in the spacetime background of a very massive object loses energy and angular momentum by emitting gravitational waves. In section 3 we investigated the characteristics of a geodesic motion in a stationary MN spacetime background. The energy $E$ and the angular momentum $L_{z}$ were regarded as constants of motion instead of adiabatically varying quantities. The accurate solution of the two body problem in the framework of general relativity is still intractable. It can only be solved numerically, although for a wide range of parameters the problem has not been adequately analyzed yet. Various approximate schemes have been proposed to compute the energy and angular momentum loss for a small mass in a Kerr metric while no other systematic way to compute such losses for other kinds of background spacetimes is available. Following the hybrid approximative method of [33] (Eqs. $(44,45)$ ), that Gair et al [16] also applied in the MN spacetime, we have computed the energy and $z$-angular momentum losses from the instantaneous geometric orbital parameters as if the spacetime were a Kerr metric. The only modification introduced by Gair et al [16] from the original hybrid model [33] was that the deviation of the quadrupole moment of the $\mathrm{MN}$ was added to the terms that are proportional to the square of the spin of the Kerr metric. We applied exactly the same modification. Then we assumed linear variations for the parameters $E, L_{z}$, that is

$$
E(t)=E(0)+\left.\frac{d E}{d t}\right|_{0} t
$$

and

$$
L_{z}(t)=L_{z}(0)+\left.\frac{d L_{z}}{d t}\right|_{0} t
$$

and we inserted them in the equations of motion, thus producing a new set of non-geodesic equations of motion that approximately describe the adiabatic inspiral. To be more specific, the energy and angular momentum losses $\left.(d E / d t)\right|_{0},\left.\left(d L_{z} / d t\right)\right|_{0}$ were computed from the semi-latus rectum and eccentricity of the geodesic motion that correspond to the initial values $E(0)$ and $L_{z}(0)$ (see Appendix $\mathrm{A}$ ). Then the non-geodesic orbit, that was created from the adiabatically varying equations of motion, was evolved for an interval of time that was long compared to all orbital periods (azimuthal and polar, e.g. $T_{\rho}$ and $T_{z}$ ), but short compared to the characteristic time of the adiabatic variations $E(0) /\left.\left(\frac{d E}{d t}\right)\right|_{0}$ and $L_{z}(0) /\left(\frac{d L_{z}}{d t}\right)_{0}$ (the latter comparison justifies the use of linear dependence for $E(t)$ and $\left.L_{z}(t)\right)$. In fact the evolution of the orbit was calculated for a time interval sufficient for the orbit to hit a resonance, cross it, and depart from it. Since the resonances we have found in exploring the geodesics in a MN metric are very thin structures, the corresponding times just to cross them were indeed very short in comparison to the characteristic time of $E$-variation and $L_{z}$-variation. In order not to waste numerical time and remain in the range of validity of the linear approximation for the adiabatic changes, we chose initial conditions for the non-geodesic orbits along the line $(\dot{\rho}=0, z=0)$ that were within a short distance away from the boundary of a resonant island itself.

The initial conditions along the aforementioned line give an adequately representative set of non-geodesic orbits to study the time it takes for the inspiraling orbits to cross through the resonant islands. Actually the non-geodesic orbits corresponding to the initial conditions that lie on the convex side of the islands (Fig. [3a) do not cross the particular resonant island; these orbits continuously recede from the islands. Thus from now on we constrain our study to non-geodesic orbits that start near the concave side of the resonant islands. On the concave side of the leftmost island there is a window of initial conditions of $\rho(0)$ along the $(\dot{\rho}=0, z=0)$ line for which the corresponding non-geodesic orbits eventually enter first this particular island of resonance. Upon its entrance a non-geodesic orbit visits all the other islands of that resonance as well. An analogous window of $\rho(0)$ s, right next to the former one, contains orbits that enter first the next island of the same resonance and so on until we reach the window that enters first the last island of the chain. After that window, it follows another window of initial conditions for which the non-geodesic orbits enter the leftmost island first. The non-geodesic orbits that start from the latter window perform an extra full rotation on the surface of section with respect to the non-geodesic orbits of the first window, before they enter the particular resonant island. During this rotation the islands move towards the center of the main island, so that they eventually encounter the evolving orbits from this new window. The succession of such windows continues, until point that the non-geodesic orbits reach the resonance is so close to the center of the main island (due the energy 
and z-angular momentum loss), that the islands have shrunk to negligible thickness and thus no resonance can be observed. However the linear approximation we use to produce the non-geodesic orbits ceases to be valid well before this happens. Therefore we have not extended our numerical computation that far.

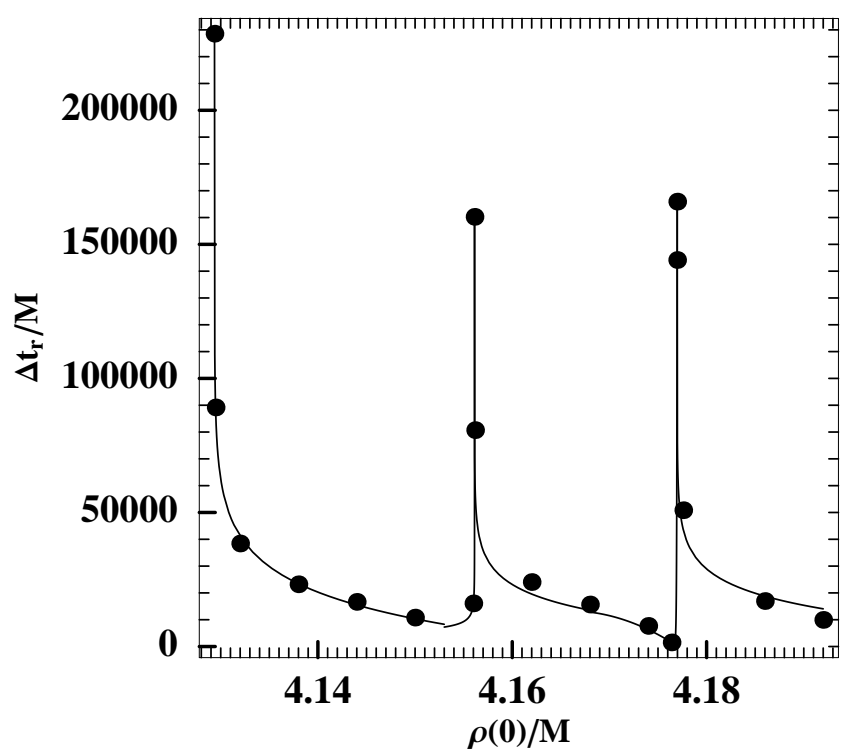

FIG. 11: The time $\Delta t_{r}$ needed by non-geodesic orbits to cross the chain of islands belonging to the $2 / 3$-resonance as a function of their initial conditions $\rho(0)$ (the initial value of the $\rho$-coordinate) along the line $\dot{\rho}=0, z=0$. The parameters used are $\mu / M=8 \times 10^{-5}, q=0.95, \chi=0.9, E(0)=0.95, L_{z}(0)=3 M$.

In order to study the observability of the resonances we have chosen a few distinct initial conditions along the above sequence and measured the time interval that the corresponding non-geodesic orbit needs to cross the $2 / 3$-resonant islands. The time $\Delta t_{r}$ spent by each non-geodesic orbits in the $2 / 3$-resonance is shown in Fig. 11 as a function of their initial conditions along the line $(\dot{\rho}=0, z=0)$. Moreover each window in the sequence of windows in Fig. 11 is demarcated by two distinct abrupt peaks of $\Delta t_{r}$ lying on either side of the window. Of course there are a number of parameters that affect this $\Delta t_{r}$ time interval, like the initial energy $E(0)$, the initial angular momentum $L_{z}(0)$ and the exact initial conditions of an orbit (in our case $\rho(0)$ ), as well as the physical characteristics of the EMRI, namely the parameters of the metric $\chi$ and $q$, and the masses of the two bodies. Especially the ratio of masses $\mu / M$ plays an important role in $\Delta t_{r}$; the lower the value of $\mu / M$ is, the longer time it takes to reach a particular island, and consequently the width of the windows is larger. Our aim is not to explore the dependence of crossing time $\Delta t_{r}$ on all these parameters, instead we explore the qualitative characteristics that relate the time of crossing with the physical characteristics of an EMRI. We observed that the exact point of entrance in the island is crucial for the time the orbit spends in that island before it exits the particular chain of islands.

In order to study how the time spent in the chain of islands depends on the entrance point we use a stroboscopic depiction, i.e. for a chain of islands of multiplicity 3 we depict only every third crossing of an orbit through a surface of section. Consequently the successive crossing points are close to each other and we can join them by a single line (thick line in Fig. 12a,b). In this figure only one island is depicted; the leftmost one of the 2/3-resonance (Fig. 3a). The stroboscopic projection of a non-geodesic orbit forms a clockwise spiral on a surface of section until it reaches the resonant island. By plotting a couple of such orbits, namely one entering the island very close to its lowest point (Fig. 12a) and a second one at a point a little higher from its lowest point and along its concave side (Fig. 12 b), we noticed that if the entrance point is near the lowest point of the island it will spend substantially more time in the resonance than when the entrance point is further upwards. In the former case (Fig. 12 $\mathrm{a}$ ) the adiabatic drift of the orbit and of the island itself forces the orbit to revolve counterclockwise three times around the island while at resonance. After these revolutions the relative drift of the orbit with respect to the island moves the orbit out of the island and the exit point is then on the right of the entrance point. In the latter case (Fig. 12p) while the orbit starts revolving counterclockwise around the island upon its entrance, it finds itself near the opposite side (the convex side) of the island when the relative drift forces the orbit to exit the island. In this case the exit point is on the left of the entrance point but very close to it. 
As mentioned before, the ratio of masses that are involved plays a radical role in the crossing time. If this ratio is $\gtrsim 10^{-4}$ the evolution of the orbit is so quick that within one $z$-period the orbit has crossed the resonance and almost no point on a surface of section is found inside the island. On the other hand if $\mu / M \lesssim 10^{-7}$ the adiabatic evolution of the orbit is so slow that the determination of the initial conditions that eventually enter an island of resonance is quite difficult due to long integration times. Interestingly enough, the range of mass ratios that lead to observable crossing times correspond to the range of masses of EMRIs for which LISA is expected to be sensitive.

(a)

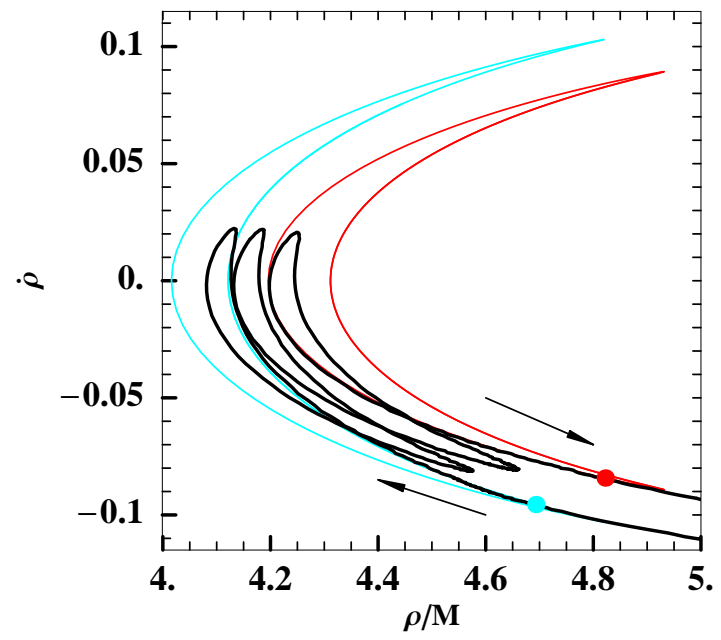

(b)

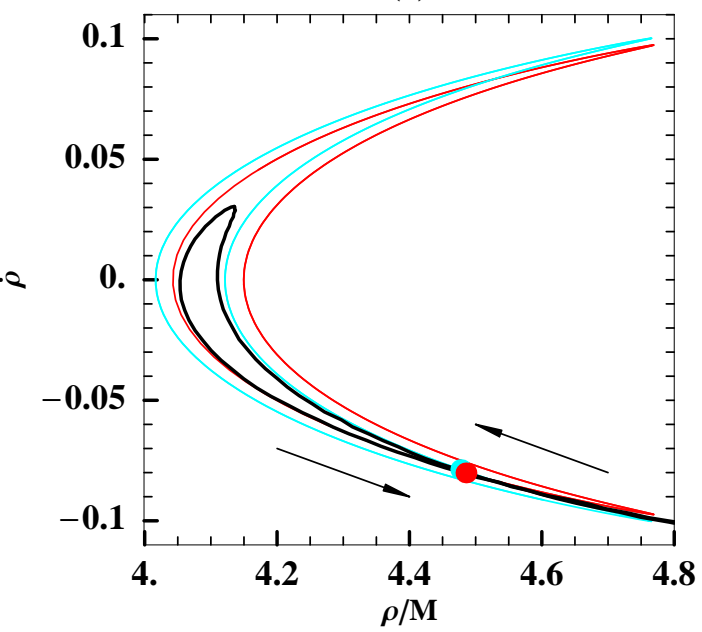

FIG. 12: The black thick lines mark the succession of every third point on the $z=0$ surface of section of a non-geodesic orbit for two different types of entrapment in the 2/3-resonance. (a) Entrapment of a non-geodesic orbit that makes 3 loops while inside the resonant island and (b) entrapment of a non-geodesic orbit that makes almost one loop. The arrows show the flow of the orbits on the $\rho, \dot{\rho}$ plane. In (a) and (b) the blue (light gray) big dot indicates the point at which the corresponding non-geodesic orbit crosses the border (blue or light gray thin line) and enters the leftmost island of the $2 / 3$-resonance, while the red (dark gray) big dot indicates the point at which the non-geodesic orbit crosses the border (red or dark gray thin line) and exits the 2/3-island of stability. Note the drift of the island during the evolution of the orbit. For both cases $\mu / M=8 \times 10^{-5}, q=0.95, \chi=0.9, E(0)=0.95, L_{z}(0)=3 M$. The non-geodesic orbits of (a) and (b) correspond to the first and the third point from the left on the diagram of Fig. 11 respectively.

The next important question one has to answer is how probable is for a non-Kerr EMRI to evolve so as to pass through a resonance during its evolution, and thus enable us to detect such a passage through a frequency analysis of the corresponding gravitational waves. Using again the MN metric as a characteristic example of a perturbed Kerr metric, we have explored how the initial conditions evolve if the orbit starts far away from a specific resonance. We have not explored all possible orbital parameters to check if they finally pass through such a resonance, since the problem depends on too many parameters: the ratio of masses $\mu / M$, the quadrupole deviation parameter $q$, the spin $\chi$, the initial orbital characteristics (the semi-latus rectum $p$, the eccentricity $e$ and the inclination of the orbit $\iota$ ) and the particular resonance we examine each time. Instead of calculating the evolution of the non-geodesic orbits for all these parameters we run an orbit with a specific initial condition to obtain a crude estimate of what are the chances to hit a resonance. More specifically we focused our attention to the resonance $2 / 3$ which according to our previous analysis has the strongest effect (the corresponding islands are the thickest ones). We started with initial values $E(0)=0.95, L_{z}(0)=3 M, q=0.95, \chi=0.9$. For these values there are two allowed regions of orbits, while the outer region is characterized by an effective potential that is qualitatively similar to the one for a Kerr metric with the same mass and angular momentum. The leftmost island of the $2 / 3$-resonance (see Fig. 3 $\mathrm{k}$ ) of such a MN spacetime then spans the interval $4.006 M \leq \rho_{2 / 3} \leq 4.109 M$.

We chose an initial orbit with the above parameters and evolved it by varying adiabatically the parameters $E, L_{z}$. The rates of their variation was computed based on the initial orbital parameters and the corresponding Kerr-like losses (see the discussion above). The surface of section of the non-geodesic orbit was drawn and the speed of the drift of the corresponding KAM curves was graphically calculated; i.e., we measured the velocity by which the leftmost part of the orbit on a surface of section corresponding to a non-geodesic orbit was moving. Due to energy and $z$-angular momentum loss the corresponding geodesic KAM curves are shrinking (circularization of the orbits). Simultaneously they are moving towards lower $\rho$-values. The net drift of the leftmost part of these geodesic curves is towards $\rho=0$ 
with a speed of the order of $\Delta \rho_{\text {orb }} / \Delta t \simeq-1.6 \times 10^{-6}$ (the minus sign means that it moves towards $\rho=0$ ). This number corresponds to a ratio of masses $\mu / M=8 \times 10^{-5}$ and initial values for the orbit $\rho(0)=4.15 M, \dot{\rho}(0)=0, z(0)=0$. Of course this drift is expected to be slower for lower $\mu / M$ values and to be slightly altered for other values of $\rho(0)$ due to changes in the corresponding orbital parameters $p, e, \iota$ that consequently induce changes in the values of $E$ and $L_{z}$ losses. On the other hand we followed the successive positions of the chain of islands for the resonance $2 / 3$ due to the values of $d E / d t, d L_{z} / d t$ used in the previous calculation. The whole chain is shrinking and approaches the center of the main island. But the center of the main island itself is moving, together with the set of islands around it, towards $\rho=0$. The net drift of the leftmost island is away from $\rho=0$ with velocity of order $\Delta \rho_{\text {isl }} / \Delta t \simeq+1.1 \times 10^{-6}$. Therefore the orbit will hit the 2/3-resonance in a time interval of order $4 \times 10^{5} \Delta \rho_{0}$, where $\Delta \rho_{0}$ is the initial distance between the value of $\rho$ for the orbit when it crosses the $z=0, \dot{\rho}=0$ surface, and the right boundary along the axis $\dot{\rho}=0$ of the leftmost $2 / 3$-resonant island.

This crude estimate for the time to hit the $2 / 3$ resonance has its own range of validity. While the orbit and the chain of islands shrink towards the center of the main island, the resonance is eventually led to disappearance. As the chain of islands approaches the central point of the main island, the islands of the $2 / 3$-resonance shrink and disappear at the center of the main island. Therefore we should not extend the estimate for the time to hit the resonance beyond the time when there are no 2/3-resonance islands at all. This upper value for the time to hit the resonance is directly related to the lowest eccentricity of orbits that will eventually hit the resonance (the closer the orbits are to the central point of the main island, the smaller is their oscillation along the $\rho$ axis). If the orbit starts with eccentricity lower than a cutoff value, the orbit will become very circular before it reaches a ratio of frequencies corresponding to the resonance of 2/3. All higher initial eccentricities, up to the eccentricity that corresponds to the initial position of the chain of islands itself will eventually cross this chain of islands, leaving a characteristic imprint on the ratio of gravitational wave frequencies. For the aforementioned initial values the least eccentric orbit that has sufficient time to hit the $2 / 3-$ resonance has initial conditions $\rho(0) \simeq 6.8 M, \dot{\rho}(0)=0, z(0)=0$ (the rotation number of the corresponding geodesic orbit is $\left.\nu_{\theta}(0)=0.26\right)$. This initial condition corresponds to orbital parameters $p(0)=9.1 M, e(0)=0.16, \iota(0)=27^{\circ}$. The time needed by such an orbit to hit the $2 / 3$-resonance is of order $5 \times 10^{5} M$, that is $\simeq 2.5\left(M / M_{\odot}\right)$ s. On the other hand the most eccentric orbit that hits the $2 / 3$-resonance in a very short time interval (since it starts right next to the concave side of the island) has orbital parameters $p(0)=7.4, e(0)=0.46, \iota(0)=19^{\circ}\left(\nu_{\theta}(0)=0.33\right)$.

Although this single numerical example represents an arbitrary initial orbital configuration, it still gives a crude estimate of the orbital characteristics that have the chance to reveal a possible non-Kerr character of the background. We expect that the value of $\mu / M$ will not alter significantly the range of orbital parameters since it will simply make the evolution faster (large $\mu / M)$ or slower (low $\mu / M)$; it will only affect the total time to hit the resonance. The initial parameters $E(0), L_{z}(0)$ will be more crucial for determining the range of parameters since they are directly related to the initial position of the islands of resonance.

\section{OBSERVATIONAL IMPRINTS}

In this section we focus our attention on the quantity that, according to our analysis, its measurement will provide us with useful information about possible deviations of an EMRI's central object from a Kerr black hole. This quantity is the ratio of the polar fundamental frequencies, that is the frequencies that characterize the oscillations of an orbit on the polar plane which rotates along with the low-mass object.

For a generic EMRI signal each fundamental frequency related to the evolution of the system, as well as any linear combination of their harmonics, will show up in its Fourier analysis. The most prominent frequency peaks $\Omega_{k}$ in the spectrum will be some integer multiples of the corresponding fundamental frequencies $\omega_{i}$; that is $\Omega_{k}=\sum_{i} m_{i}^{(k)} \omega_{i}$, where $m_{i}^{(k)}$ are integers. Therefore the ratio of the frequencies $\Omega_{k}$ of the most intense peaks that are observed in the Fourier spectrum of the signal and are related to polar oscillations, but which are not harmonically related to each other, will be of the form

$$
\frac{\Omega_{\rho}}{\Omega_{z}}=\frac{m_{1} \nu_{\theta}+m_{2}}{n_{1} \nu_{\theta}+n_{2}},
$$

with $m_{1}, m_{2}, n_{1}, n_{2}$ some integers. In our case the highest peaks in the Fourier spectrum of the two polar coordinates yield a ratio of Fourier frequencies equal to $\left(\nu_{\theta}-1\right) / \nu_{\theta}\left(m_{1}=1, m_{2}=-1, n_{1}=1, n_{2}=0\right)$.

The Fourier spectrum of the coordinates used to describe the polar oscillations consists of both fundamental frequencies and combinations of their harmonics, as mentioned before. This may render the determination of Fourier frequencies problematic since we practically analyze a finite length of an orbit that leads to Fourier peaks of finite width; thus two frequency peaks that are close to each other may not be easily discerned. In order to overcome this 
(a)

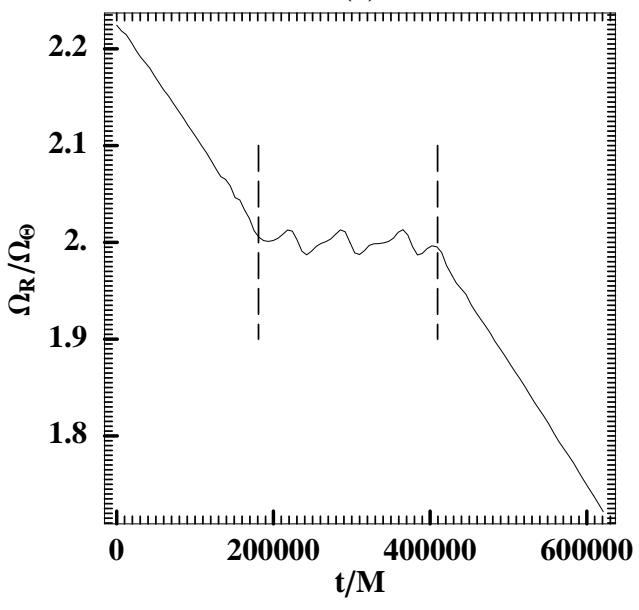

(b)

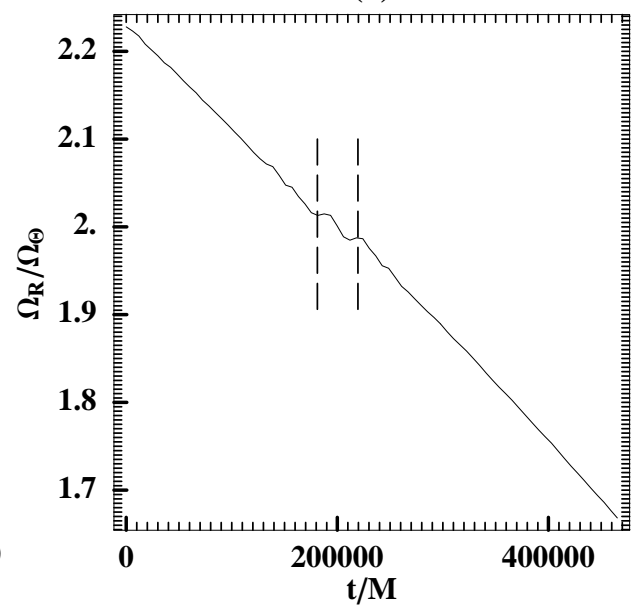

FIG. 13: The evolution of the ratio $\Omega_{R} / \Omega_{\Theta}$ as function of the coordinate time $t$ for (a) the non-geodesic orbit shown in Fig. 12 a and (b) the non-geodesic orbit shown in Fig. 12 b. The vertical dashed lines demarcate the time intervals that the non-geodesic orbit spends in the interior of the $2 / 3$-resonance.

problem, we used the coordinates $R$ (see Eq. (5k) ) and

$$
\Theta=\tan ^{-1}\left(\frac{z}{\rho}\right)
$$

instead of the $\rho, z$ coordinates. As shown in Appendix B the new coordinates are much better in order to reveal their frequency content with good accuracy. Thus the frequencies used in the following paragraphs have been computed by Fourier analyzing the time series of the orbits described by these "cleaner" coordinates $R$ and $\Theta$.

As explained in Sec. 3.1, if an EMRI source evolves in the gravitational field of a massive object that is similar to, but not exactly a Kerr black hole, it has good chances to pass through a discernible resonance -like the 2/3-resonance, for which some quantitative estimates have been given in the previous section. If this happens within the range of distances that a gravitational-wave detector like LISA is sensitive to detect a corresponding signal [3], we should be able to observe the non-Kerr character of the central object by tracking a transient stationary value of the ratio of frequencies $\nu_{\theta}$.

We have evolved a few orbits that initially lie near a resonance. We have avoided to investigate orbits that start far away from a resonance since this would be quite expensive numerically. Also the time until the inspiraling orbit hits a resonance is of no special interest since the evolution of the orbits outside a resonance is expected to be quite similar both in a Kerr and in a perturbed Kerr metric; in both cases the ratio of frequencies will vary monotonically with time.

As explained in Sec. 4 the passage through a resonance depends crucially on the location of the entrance point in an island of resonance. Therefore we will present two graphs that depict the evolution of the ratio of frequencies as a function of time. The first one corresponds to an entrance point, such that the orbit gets trapped for a few circles in the islands of the 2/3-resonance. The second one corresponds to an entrance point, such that the orbit performs almost one loop and then departs from the chain of islands. The two cases are exactly the ones that were presented in Sec. 4 when we discussed the duration of crossing a resonance as a function of the initial conditions (cf. Fig. 12a and $\mathrm{b}$ ). In both cases the orbits have been evolved for sufficiently long time before and after their entrance, so that the different type of evolution of the ratio of frequencies then is clear.

In Fig. 13 we have plotted the ratio of frequencies (i) for an orbit that its surface of section evolves through an island of 2/3-resonance according to Fig. 12 and (ii) for an orbit that corresponds to Fig. 12b. The orbits have been divided in time segments of length $\Delta t \simeq 5000 \mathrm{M}$. Each such segment of the orbit is Fourier analyzed and the frequencies $\Omega_{R}, \Omega_{\Theta}$ are recorded (see discussion above). Finally the ratio of these frequencies is plotted as a function of $t$. For the case in Fig. 12 a we have plotted also the evolution of the two frequencies (Fig. 114). In this figure we have highlighted the crossing of the 2/3-resonance by the non-geodesic orbit by scaling accordingly the frequency $\Omega_{R}$ so that the two frequencies coincide when they are superimposed. As shown in Figs. 13 the ratio of frequencies evolves quite differently while the non-geodesic orbit passes through a resonance compared to its behavior when this orbit moves adiabatically from one KAM curve to another (outside the resonance). However the ratio of frequencies 
does not have the form of a pure plateau when at resonance. Instead the ratio of frequencies forms a number of oscillations (the number of cycles matches the number of loops that the orbit performs while it crosses a particular island) superimposed on a plateau. These oscillations are mainly due to the finiteness of the orbit that is Fourier analyzed. In most of the cases the orbit performs only part of a loop inside an island though (Fig. 12 $\mathrm{p}$ ). Then there is only a fraction of an oscillation apparent on the evolution of $\Omega_{R} / \Omega_{\Theta}$ and the variation of the curve may not show very clearly the underlying plateau (Fig. 13b). A possible way to discern the plateau in these cases is by extrapolating the lines that describe the variation of $\Omega_{R} / \Omega_{\Theta}$ before and after the plateau and look for some finite distance between these lines. This distance will be a measure of the time spent within a resonance and should be related to the characteristics of the source.

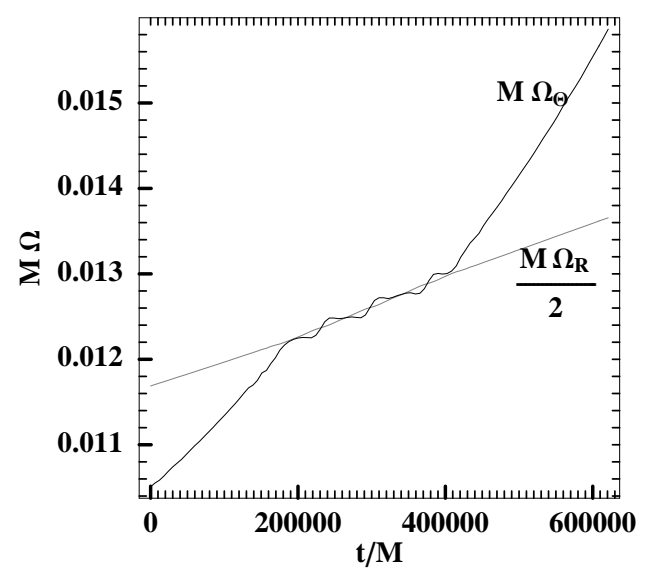

FIG. 14: The evolution of the two polar frequencies $\Omega_{R}$ and $\Omega_{\Theta}$ as functions of the coordinate time $t$ for the non-geodesic orbit shown in Fig. 12 before, during and after the crossing of the $2 / 3$-resonance

\section{CONCLUSIONS}

In this paper we have investigated what are the manifestations of EMRIs consisting of a small mass compact object that is orbiting around a much more massive compact object that is not an exact Kerr black hole. Our study has been based on a generic qualitatively new feature that discerns an integrable Hamiltonian system from a system that slightly deviates from an integrable one; namely, the appearance of Birkhoff chains of islands on a Poincaré surface of section, instead of a set of fixed points of the corresponding integrable system. We have chosen a specific exact solution of the vacuum Einstein equations, a member of the family of the Manko-Novikov metrics, which has been extensively studied in the literature. This MN metric is characterized, besides its mass $M$ and spin $S$, by one more parameter $q$, which measures the deviation of its quadrupole moment from the corresponding Kerr (the one with the same mass $M$ and spin $S$ ). Thus if the $q$ parameter is set to zero the Manko-Novikov metric turns into an exact Kerr metric. We have used this metric as a generic example of a slightly non-Kerr metric.

By studying first the geodesic orbits in a MN background, we showed that when the orbit hits a resonance, that is when the ratio of the frequencies $\omega_{\rho}, \omega_{z}$ that characterize the orbital oscillations on the polar plane (the plane that passes through the axis of symmetry of the central object and rotates along with the low mass object) is a rational number, then the orbit exhibits a qualitatively new behavior: for a finite range of initial conditions the ratio of these frequencies is constant, in contrast to what happens in the integrable case of a Kerr metric. Actually we have found two such chains of Birkhoff islands in the most interesting region of bound orbits (the outer one), which correspond to the resonances $2: 3$ and 1:2 respectively. Furthermore we explored the thickness and the location of the $2 / 3$ islands with respect to the various parameters that characterize the metric and the parameters describing the orbits. Thus, in a realistic EMRI case with a non-Kerr central object, while the orbit of the low-mass object evolves adiabatically, the ratio between the corresponding frequencies is expected to remain constant (the two frequencies get locked to each other) for a finite time interval, exhibiting a characteristic plateau in the evolution of this ratio.

Although there is a wide range of time intervals corresponding to such a plateau, depending on the exact parameters of the metric, the ratio of masses, and the specific parameters and initial value of the coordinates of the orbit, the appearance of a plateau in the evolution of the ratio of frequencies with time is a generic feature. Moreover the most 
prominent plateaus correspond to fractions of small integer numbers, since these are related to strong resonances, and thus they are easier to investigate through focused data analysis of the gravitational-wave signals from EMRI sources. We found that the duration of the plateau through the resonance of $2: 3$ is roughly of the order of $\Delta t_{r} \approx 0.15\left(M / M_{\odot}\right) \mathrm{s}$ for a ratio of masses $\mu / M=8 \times 10^{-5}$ and a MN metric with $q=0.95$ and $\chi=0.9$. This time $\Delta t_{r}$ increases for lower ratios of masses and decreases for higher ratios of masses. For $\mu / M \gtrsim 10^{-4}$ the evolution of the orbit through a resonance is so quick that the corresponding plateau is rather impossible to be detected though.

The initial orbital parameters (semi-latus recta $p$, eccentricities $e$, and inclinations $\iota$ ) that will eventually lead the orbit through a strong resonance apparently span quite a wide range; hence a high fraction of possible sources for LISA are expected to exhibit a plateau if they involve such a non-Kerr central object. A rough estimate yields $e$ 's, and $\iota$ 's in the range of $0.17-0.45$ and $44^{\circ}-19^{\circ}$ respectively, corresponding to initial semi-latus recta of the order $7.4 M-9.1 M$, that will evolve so as to hit the resonance $2: 3$.

We believe that a focused data analysis of signals of LISA in the temporal region where the fundamental frequencies related to the orbital oscillations on the polar plane of the EMRI's orbit has high chances to reveal or at least constrain the non-Kerrness of the EMRI central object. This analysis, along with a number of other tests that have been proposed by other people [8, 14, 16], and are related with the non-Kerrness of the metric involved, could enhance our knowledge about the astrophysical processes that lead to creation of ultra-compact supermassive objects at the centers of galaxies.

\section{Acknowledgments}

We would like to thank Mr. John Deligiannis for an independent cross-checking of our first numerical results. G. Lukes-Gerakopoulos was supported in part by the I.K.Y. scholarships and by the Research Committee of the Academy of Athens. T. Apostolatos would like to thank K.S. Thorne and I. Mandel for very enlightening discussions on the subject. Also T. Apostolatos acknowledges the research funding program "Kapodistrias" of ELKE (Grant No 70/4/7672) and the I.K.Y. (IKYDA 2010)

\section{Appendix A: Computation of the orbital parameters}

In order to compute the energy and z-angular momentum losses of the EMRI due to gravitational radiation we have used the hybrid model of Gair and Glampedakis [33] which have actually been constructed to measure the corresponding fluxes at infinity of an EMRI in a Kerr background. These instantaneous fluxes, described by formulae $(44,45)$ of their paper, are given as functions of the orbital parameters $p, e, \iota$ of the corresponding geodesic orbit of a test particle in a Kerr metric. When the geodesic orbit is described in Boyer-Lindquist coordinates the aforementioned orbital parameters are given by analogy with the Keplerian quantities:

$$
\begin{aligned}
& p=\frac{2 r_{+} r_{-}}{r_{+}+r_{-}}, \\
& e=\frac{r_{+}-r_{-}}{r_{+}+r_{-}}, \\
& \iota=\frac{\theta_{+}-\theta_{-}}{2},
\end{aligned}
$$

where $r_{+}\left(r_{-}\right)$are the maximum (minimum) radial values, while $\theta_{+}\left(\theta_{-}\right)$are the maximum (minimum) values of $\theta$ coordinate along the orbit.

The MN metric, and consequently the geodesic orbits that we computed numerically, are expressed in cylindrical coordinates. Thus in order to estimate the orbital parameters, we have transformed the $\rho, z$ coordinates in BoyerLindquist coordinates (the coordinates in which the Kerr metric that we obtain when we set $q=0$ in our MN metric yields its usual form [34]) according to

$$
r / M=1+\sqrt{-\sigma_{2}+\sqrt{\sigma_{2}^{2}+\sigma_{1} *(z / M)^{2}}}, \quad \theta=\cos ^{-1}\left(\frac{z / M}{(r / M)-1}\right),
$$

where

$$
\sigma_{1}=\chi^{2}-1, \quad \sigma_{2}=\frac{\sigma_{1}-(\rho / M)^{2}-(z / M)^{2}}{2}
$$




\section{Appendix B: Better coordinates for Fourier analysis}

The gravitational waves from an EMRI source are expected to have the same Fourier spectrum as the orbit itself. Thus by monitoring a gravitational wave from such a source we can reveal all the frequencies that are related with the orbit. By using a specific set of coordinates to describe the orbit we may face the following problem: Each coordinate most probably will describe a synthesis of all oscillations. This will cause problems in the Fourier analysis of the coordinate itself, because the sample that is analyzed has finite length as it is the case when the adiabatically inspiraling orbit in a MN is Fourier analyzed (see Sec. 51).

(a)

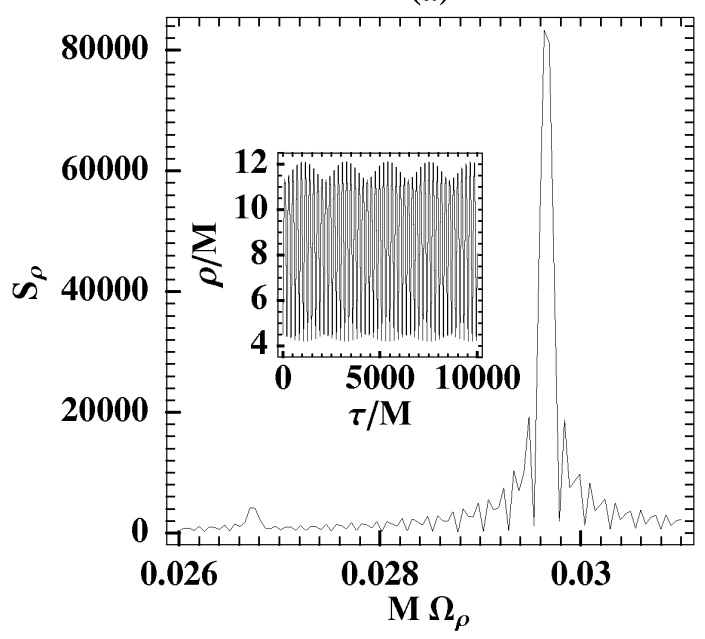

(b)

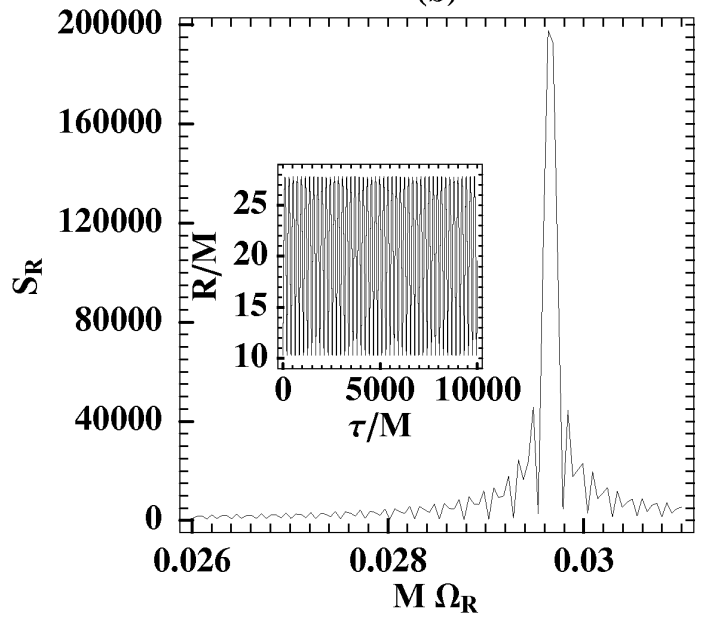

FIG. 15: (a) The Fourier spectrum of the $\rho$-coordinate for a geodesic orbit. Embedded in (a) is the evolution of the $\rho$ coordinate along the proper time that produces this Fourier spectrum. The side frequency is apparent as a small secondary peak on the left of the fundamental frequency peak. The data-series are quite long here; namely $\Delta \tau_{\text {tot }}=5 \times 10^{4} M$. However small the secondary peak may be, it causes significant problems when one tries to determine the fundamental frequencies of the adiabatically varying orbit by analyzing a data-series 10 times shorter in order to monitor the adiabatic evolution of the frequencies. In that case the fundamental-frequency peak is 10 times broader and the secondary peak slightly alters its shape. (b) The Fourier spectrum of the $R$-coordinate for the same geodesic orbit as in (a). Embedded in (b) is the evolution of the $R$-coordinate along the proper time that produces this Fourier spectrum. The $R$-coordinate has practically no modulation and the corresponding Fourier spectrum has no secondary peaks.

When the cylindrical coordinates are used to describe the orbital oscillations on the polar plane there is a large uncertainty in determining the fundamental Fourier frequencies of the corresponding coordinates. This is due to the fact that these coordinates exhibit significant amplitude modulation. This modulation causes the appearance of side-frequencies which may render problematic the determination of the fundamental frequencies of a finite-length data series (see Fig. 15). We found that the analysis is much clearer in a new set of coordinates:

$$
R=\frac{\sqrt{\rho^{2}+z^{2}}}{k}, \Theta=\tan ^{-1}\left(\frac{z}{\rho}\right) .
$$

Thus we transformed the $\rho, z$ coordinate into $R, \Theta$ coordinates before we Fourier analyze them, and we got a much clearer picture of the evolution of the ratio of frequencies in the adiabatically changing orbits.

[1] Reil K., the Ligo and Virgo Scientific Collaborations, J. Phys.: Conf. Ser., 203, 012002 (2010)

[2] Abbott B.P. et al, arXiv:0909.3583 (2009)

[3] P. Bender,P. Danzmann, and the LISA Study Team (1998) "Laser Interferometer Space Antenna for the Detection of Gravitational Waves, Pre-Phase A Report" MPQ 233 (Garching: Max-Planck-Institüt für Quantenoptik)

[4] Gair J., Class. Quantum Grav., 26, 094034 (2009) 
[5] Preto M., Amaro-Seoane P. Ap. J.., 708, L42 (2010)

[6] Amaro-Seoane P, Gair J R, Freitag M, Miller M C, Mandel I, Cutler C J and Babak S, Class. Quantum Grav., 24, R113 (2007).

[7] Barack L., Cutler C., Phys. Rev. D, 75, 042003 (2007)

[8] Glampedakis, K., Babak, S., Class. Quantum Grav., 23, 4167 (2006)

[9] Babak, S., Gair J., Porter E. Class. Quantum Grav., 26, 135004 (2009)

[10] Barack L., Cutler C., Phys. Rev. D, 70, 122002 (2004)

[11] Cornish, N. J., Porter E. K., Class. Quantum Grav., 24, S501 (2007)

[12] Ryan F. D., Phys. Rev. D, 52, 5707 (1995)

[13] Ryan F. D., Phys. Rev. D, 56, 1845 (1997)

[14] Collins, N. A., Hughes, S. A., Phys. Rev. D, 69, 124022 (2004)

[15] Barausse, E. , Rezzolla L., Petroff D., Ansorg M., Phys. Rev. D, 75, 064026 (2007)

[16] Gair J.R., Li C., Mandel I., Phys. Rev. D, 77, 024035, (2008)

[17] Manko V.S., Novikov I.D., Class. Quant. Grav., 9, 2477, (1992)

[18] Carter B., Phys. Rev., 174, 1559 (1968)

[19] Chandrasekhar S., 'The mathematical theory of Black holes', Oxford University Press, (1992)

[20] Kolmogorov A. N., Dokl. Akad. Nauk. SSSR, 98, 527 (1954); Arnold V. I., Russian Math. Survey, 18, 13-40, 85-191 (1963); Moser J., Nachr. Akad. Wiss. Gttingen Math.-Phys. Kl. II, 1-20, (1962)

[21] Apostolatos T.A., Lukes-Gerakopoulos G., Contopoulos G. Phys. Rev. Lett., 103, 111101, (2009)

[22] Castejon-Amenedo J., Manko V. S., Phys. Rev. D, 41, 2018, (1990)

[23] O'Neil B., 'The Geometry of Kerr Black Holes', A. K. Peters, (1995)

[24] Lichtenberg A. J. , Lieberman M. A. , 'Regular and Chaotic Dynamics', Springer-Verlag, (1992)

[25] Poincaré H., Rend. Circ. Mat. Palermo, 33, 375 (1912); Birkhoff G. D., Trans. Am. Math. Soc. , 14, 14 (1913)

[26] Rosenbluth M. N., Sagdeev R. Z., Taylor J. B., Zaslavsky G. M., Nucl. Fusion , 6, 297-300, (1966); Contopoulos G., 'Les Nouvelles Méthodes de la Dynamique Stellaire', 223 (1966); Bull. Astron., 2, 223 (1967); Zaslavsky G. M., Chirikov B. V., Sov. Phys. Usp., 14, 549 (1972)

[27] Chirikov B. V., Phys. Rep. , 52, 263, (1979)

[28] Contopoulos G., 'Order and chaos in dynamical Astronomy',, Springer (2002)

[29] Laskar J., Celest. Mech. Dynam. Astron.,56, 191, (1993)

[30] Voglis N., Efthymiopoulos C., J. Phys. A: Math. Gen.,31, 2913, (1998)

[31] Schmidt W., Class. Quant. Grav., 19, 2743, (2002)

[32] Contopoulos G., Astron. J.,76, 147, (1971)

[33] Gair J. ,Glampedakis K. , Phys. Rev. D, 73, 064037 (2006).

[34] Misner C., Thorne K.S., Wheeler A.J., 'Gravitation', W. H. Freeman and Company (1970) 